\title{
N-3 Long-Chain Polyunsaturated Fatty Acids, Eicosapentaenoic and Docosahexaenoic Acid, and the Role of Supplementation during Cancer Treatment: A Scoping Review of Current Clinical Evidence
}

\author{
Marnie Newell ${ }^{1}\left(\mathbb{D}\right.$, Vera Mazurak ${ }^{1}$, Lynne M. Postovit ${ }^{2,3}$ and Catherine J. Field ${ }^{1, *(D)}$ \\ 1 Department of Agricultural, Food and Nutritional Science, Faculty of Agricultural, Life and Environmental \\ Sciences, University of Alberta, Edmonton, AB T6G 2E1, Canada; marnie.newell@ualberta.ca (M.N.); \\ vmazurak@ualberta.ca (V.M.) \\ 2 Department of Oncology, Faculty of Medicine and Dentistry, University of Alberta, Edmonton, \\ AB T6G 2R7, Canada; postovit@ualberta.ca \\ 3 Department of Biomedical and Molecular Sciences, Queen's University, Kingston, ON K7L 3N6, Canada \\ * Correspondence: catherine.field@ualberta.ca; Tel.: +1-(780)-492-2597
}

check for updates

Citation: Newell, M.; Mazurak, V.; Postovit, L.M.; Field, C.J. N-3

Long-Chain Polyunsaturated Fatty

Acids, Eicosapentaenoic and

Docosahexaenoic Acid, and the Role of Supplementation during Cancer Treatment: A Scoping Review of Current Clinical Evidence. Cancers 2021, 13, 1206. https://doi.org/ 10.3390/cancers13061206

Academic Editor: Xianjun Fang

Received: 10 February 2021

Accepted: 6 March 2021

Published: 10 March 2021

Publisher's Note: MDPI stays neutral with regard to jurisdictional claims in published maps and institutional affiliations.

Copyright: (C) 2021 by the authors Licensee MDPI, Basel, Switzerland. This article is an open access article distributed under the terms and conditions of the Creative Commons Attribution (CC BY) license (https:/ / creativecommons.org/licenses/by/ $4.0 /)$.
Simple Summary: There has been extensive research into the beneficial anticancer effects of n-3 longchain polyunsaturated fatty acids (LCPUFA), eicosapentaenoic acid (EPA) and docosahexaenoic acid (DHA) in preclinical models of cancer. However, clinical evidence is limited. The aim of this scoping review was to summarize the current clinical evidence of n-3 LCPUFA supplementation in cancer treatment and highlight areas where more clinical evidence is needed. We summarized the results of 57 clinical trials with an EPA/DHA intervention and determined that supplementation could improve a variety of outcomes important to the patient and the disease process, including immune system modulation, improved weight maintenance and increased disease-free or progression-free survival. There is, however, a need for larger, well-controlled, statistically powered randomized controlled trials to move $\mathrm{n}-3$ supplementation to clinical practice.

Abstract: This scoping review examines the evidence for $n-3$ long-chain polyunsaturated fatty acid [LCPUFA, eicosapentaenoic acid (EPA) and docosahexaenoic acid (DHA)] supplementation in clinical cancer therapy. A comprehensive literature search was performed to identify relevant clinical intervention studies conducted through August 2020. Fifty-seven unique cancer trials, assessing EPA and/or DHA supplementation pre- or post-treatment, concomitant with neoadjuvant chemotherapy, radiation or surgery, or in palliative therapy were included. Breast, head and neck, gastrointestinal, gastric, colorectal/rectal, esophageal, leukemia/lymphoma, lung, multiple myeloma and pancreatic cancers were investigated. Across the spectrum of cancers, the evidence suggests that supplementation increased or maintained body weight, increased progression-free and overall survival, improved overall quality of life, resulted in beneficial change in immune parameters and decreased serious adverse events. Taken together, the data support that EPA and/or DHA could be used to improve outcomes important to the patient and disease process. However, before incorporation into treatment can occur, there is a need for randomized clinical trials to determine the dose and type of n-3 LCPUFA intervention required, and expansion of outcomes assessed and improved reporting of outcomes.

Keywords: docosahexaenoic acid (DHA); eicosapentaenoic acid (EPA); clinical; intervention; immune; outcomes

\section{Introduction}

In 2020, an estimated 19.3 million cases of cancer were diagnosed worldwide. The most frequently diagnosed cancers across both sexes was breast $(11.7 \%)$, followed by lung 
(11.4\%), colorectal (10\%) and prostate cancer (7.3\%) [1]. Despite advances in diagnosis and treatment, cancer accounted for an estimated 10 million deaths globally in 2020 [1]. Cancer is the second leading cause of death in the United States [2] and the leading cause of death in Canada [3]. Improving current conventional therapies and treatment paradigms could result in improved patient outcomes and a reduction in deaths. As such, there has been extensive research into the efficacy of n-3 long-chain polyunsaturated fatty acids (LCPUFA), eicosapentaenoic acid (EPA) and docosahexaenoic acid (DHA) in preclinical models of cancer. The pleiotropic effects of n-3 LCPUFA on tumors include increasing apoptosis, inducing cell cycle arrest, decreasing cell growth, and halting proliferation in experimental models of cancer (reviewed in [4-6]).

While there is a growing body of strong preclinical evidence, evidence in patient populations is limited. The difficulty in translating laboratory findings to a clinical setting arises from tremendous heterogeneities that exist within tumors (intra-tumoral) and between patients which cannot readily be replicated in immortalized cancer cell models. Moreover, the role of the tumor microenvironment, including cells of the immune system is difficult to assess in immunocompromised animal models. Furthermore, side effects from cancer and the treatment for cancer have not been properly assessed in animal models. This includes pain, weight loss, quality of life (QOL) and peripheral neuropathy or other chemotherapyspecific adverse effects. The aim of this scoping review is to examine the current clinical evidence on n-3 LCPUFA supplementation in cancer treatment and highlight areas where more clinical evidence is needed.

\section{Materials and Methods}

\subsection{Study Inclusion and Exclusion Criteria}

Studies from peer-reviewed literature were included if they involved cancer patients where n-3 LCPUFAs (EPA and/or DHA) were provided as the intervention during cancer treatment. Review papers, abstracts, individual case reports and animal or cell line studies were excluded.

\subsection{Data Sources, Search Strategy and Study Selection}

A systematic literature search strategy was used to identify relevant articles. We searched electronic bibliographic databases including Ovid MEDLINE and EBSCOhost CINAHL from each database inception through August 2020. Searches were restricted to peer-reviewed studies. Search terms were searched as MeSH headings or keywords in title or abstract and were derived from (1) DHA/EPA/dietary fats (2) immune response/inflammation and (3) cancer/solid tumors/oncology treatment. Titles and abstracts of all citations were screened based on predefined eligibility and duplicates were removed. Full texts of eligible abstracts were exported to EndNote and underwent a second screen by two investigators and were verified for inclusion eligibility.

\subsection{Data Extraction and Synthesis}

Data extraction was completed by $\mathrm{MN}$ and reviewed for accuracy by the primary investigator (CJF). Data extraction included manuscript information (authors, title, year of publication), study characteristics (primary objective, study design, cancer localization and stage), patient characteristics [number enrolled/completed, mean age, gender, ethnicity, body weight/body mass index (BMI)] intervention characteristics (chemotherapy/radiation/surgical treatment, treatment length, n-3 LCPUFA type: capsules, oral supplementation, enteral/parenteral nutrition, intervention length relative to treatment length, frequency of administration, control characteristics, assessment of compliance), and outcome characteristics (quantitative and qualitative assessments). 


\section{Results}

\subsection{Study Selection}

The initial database searches yielded 729 distinct citations from Ovid MEDLINE and 237 from EBSCOhost CINAHL. Twenty papers were found to be duplicates and removed. After evaluation of the titles and abstracts, an additional 707 articles were excluded for failing to meet the inclusion and exclusion criteria. After screening the abstracts based on the eligibility criteria, 147 papers were exported from Ovid MEDLINE and 92 from EBSCOhost CINAHL, resulting in a total of 239 articles. An additional 182 articles were excluded as they failed to meet the criteria resulting in a final number of 57 studies included in the current review (Figure 1).

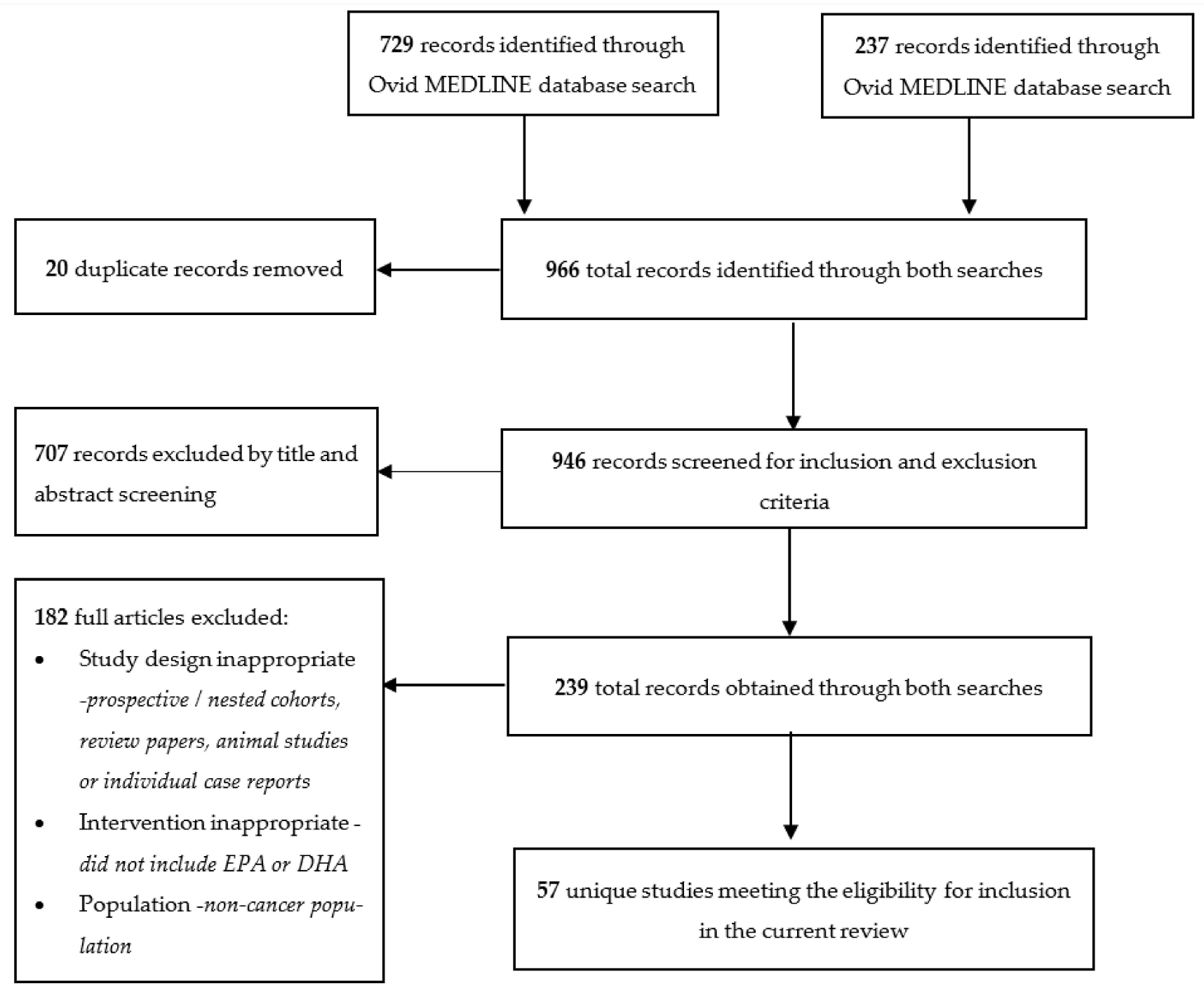

Figure 1. Flow chart of search and screening results for review inclusion.

\subsection{Overview of Studies}

A spectrum of cancers including breast [7-16], head and neck [15,17-19], gastrointestinal [14,20-27], gastric [21,28-31], colorectal/rectal [21,32-45], esophageal [15,19,21,46,47], leukemia/lymphoma [48], lung [14,15,21,40,49-55], multiple myeloma [56] and pancreatic $[14,21,55,57-63]$ have been investigated for efficacy of $n-3$ supplementation pre- or post-treatment [12,13], concomitant with neoadjuvant chemotherapy or radiation $[7-12,15,16,18-21,26,27,30-34,37,45,46,48-51,53,54,56,62-64]$, in conjunction with 
surgery $[17,18,22-25,28,35,38,42,44,46]$ or during palliative therapy $[14,36,39-41,47,52,57-61,64,65]$. Furthermore, DHA and EPA treatments in a clinical setting have been delivered through multiple modalities including capsules: Tables 1 and 2 [7-14,16,20,26,27,30,32-36,45,48-52,56-58,63], oral: Table $3[15,17,18,21,31,37-41,53-55,59-61,64]$ and enteral/parenteral supplementation: Table 4 [19,22-25,28,29,42-44,46,47,62,65]. Much of the current body of research has occurred in surgical or palliative patients receiving oral or enteral/parenteral nutrition that is routinely employed in cancer patients to provide nutritional support, especially in instances of weight loss and cachexia or during surgical interventions.

Table 1. Randomized controlled trials providing N-3 capsule supplementation concomitant with chemotherapy.

\begin{tabular}{|c|c|c|c|c|c|c|c|}
\hline $\begin{array}{c}\text { Cancer Type } \\
\text { (Stage) }\end{array}$ & $\begin{array}{c}\mathbf{N} \\
(\text { Int/CNT) } \\
\text { Female/Male }\end{array}$ & $\begin{array}{c}\text { Age, Body } \\
\text { Weight and } \\
\text { BMI } \\
\text { (Int/CNT) }\end{array}$ & Chemotherapy & $\begin{array}{c}\text { N-3 }(\mathrm{g} \\
\text { Total/Day = } \\
\text { EPA/DHA) } \\
\text { CNT }\end{array}$ & $\begin{array}{l}\text { Treatment } \\
\text { Duration }\end{array}$ & $\begin{array}{l}\text { Experimental } \\
\text { Findings }\end{array}$ & Ref \\
\hline $\begin{array}{c}\text { Breast } \\
\text { (metastatic) }\end{array}$ & $25 \mathrm{~F}$ & $\begin{array}{c}\text { Age }=58 \\
(32-71)\end{array}$ & $\begin{array}{l}\text { Cyclophosphamide, } \\
\text { Fluorouracil, } \\
\text { Epirubicin }\end{array}$ & $\begin{array}{c}1.8 \mathrm{~g} \text { DHA } \\
\mathrm{CNT}=\mathrm{N} / \mathrm{A}\end{array}$ & 18 weeks & $\begin{array}{c}\text { Stratified by } \\
\text { amount of DHA } \\
\text { incorporated into } \\
\text { plasma } \\
\uparrow \text { DHA group } \\
\text { associated with } \\
\text { longer time to } \\
\text { progression ( } 8.7 \\
\text { months vs. } 3.5 \\
\text { months); } \uparrow \text { OS (34 } \\
\text { vs. } 18 \text { months; } \downarrow \\
\text { neutropenia, } \\
\text { anaemia and } \\
\text { thrombopenia }\end{array}$ & 2009 [7] \\
\hline Breast & $57 \mathrm{~F}(30 / 27)$ & $\begin{array}{c}\text { Age }=46.2 \pm \\
9.8 / 45.7 \pm 12.0 \\
\mathrm{BMI}=46.0 \pm \\
9.0 / 44.1 \pm 8.9\end{array}$ & Paclitaxel & $\begin{array}{l}1.2 \mathrm{~g}=0.19 \mathrm{~g} \\
\text { EPA } / 1.0 \mathrm{~g} \\
\text { DHA } \\
\text { CNT }= \\
\text { sunflower oil }\end{array}$ & $\begin{array}{l}4 \text { cycles }+1 \\
\text { month post }\end{array}$ & $\begin{array}{c}\text { N-3: } 70 \% \downarrow \text { risk of } \\
\text { peripheral } \\
\text { neuropathy } \\
\text { incidence }\end{array}$ & $\begin{array}{c}2012 \\
{[9]}\end{array}$ \\
\hline $\begin{array}{l}\text { Breast } \\
\text { (I-III) }\end{array}$ & 209 F (102/107) & $\begin{array}{c}\text { Age }= \\
59.5 / 59.1 \\
\text { Body weight = } \\
79.0(77.3-79.8)\end{array}$ & $\begin{array}{l}\text { Anastrozole, } \\
\text { Exemestane or } \\
\text { Letrozole }\end{array}$ & $\begin{array}{c}3.3 \mathrm{~g}=2.24 \mathrm{~g} \\
\mathrm{EPA} / 1.12 \mathrm{~g} \\
\mathrm{DHA} \\
\mathrm{CNT}= \\
\text { soybean and } \\
\text { corn oil }\end{array}$ & 24 weeks & $\begin{array}{c}\text { Both groups: } \downarrow \text { in } \\
\text { pain symptoms in } \\
\text { but no proof of n-3 } \\
\text { efficacy; when } \\
\text { stratified by BMI, } \\
\text { n-3 significantly } \downarrow \\
\text { pain in obese } \\
\text { patients }\end{array}$ & $\begin{array}{c}2015 \\
{[10,11]}\end{array}$ \\
\hline $\begin{array}{l}\text { Breast } \\
\text { (IIIB) }\end{array}$ & $\begin{array}{c}48 \mathrm{~F} \\
(24 / 24)\end{array}$ & $\begin{array}{c}\text { Age }=46.5 \pm \\
8.1 / 48.5 \pm 8.8\end{array}$ & $\begin{array}{l}\text { Cyclophosphamide, } \\
\text { Doxorubicin, } \\
\text { Fluorouracil }\end{array}$ & $\begin{array}{l}1.0 \mathrm{~g} \mathrm{~N}-3 \\
\mathrm{CNT}= \\
\text { unknown } \\
\text { source }\end{array}$ & 51 days & $\begin{array}{c}\text { N-3: } \downarrow \text { Ki67 }(39.2 \pm \\
5.3 \text { vs. } 42.4 \pm 4.8, \mathrm{P} \\
=0.03), \downarrow \text { VEGF } \\
(29.5 \pm 5.4 \text { vs. } 32.7 \\
\pm 5.2, \mathrm{P}=0.04) . \uparrow \\
\text { OS }(30.9 \pm 3.7 \text { vs. } \\
25.9 \pm 3.6 \text { weeks, P } \\
=0.05 ; \mathrm{HR}=0.41, \\
95 \% \text { CI: } 0.20-0.84 \\
\text { and } \uparrow \text { DFS }(28.5 \pm \\
\text { 3.3 vs. } 23.7 \pm 3.6, \mathrm{P} \\
=0.03 ; \mathrm{HR}=0.44, \\
\text { 95\% CI: } 0.22-0.87\end{array}$ & $\begin{array}{c}2019 \\
{[8]}\end{array}$ \\
\hline $\begin{array}{l}\text { Breast } \\
\text { (I-II) }\end{array}$ & $5 \mathrm{~F}$ & $\begin{array}{c}\text { Age }=50 \\
(34-60)\end{array}$ & $\begin{array}{l}\text { Cyclophosphamide } \\
+ \text { Fluorouracil + } \\
\text { Doxoru- } \\
\text { bicin/Adriamycin } \\
\text { or Paclitaxel }\end{array}$ & $\begin{array}{c}1.2-1.8 \mathrm{~g}= \\
0.72 \mathrm{~g}-1.1 \mathrm{~g} \\
\text { EPA } / 0.48-0.72 \\
\text { g DHA }\end{array}$ & 130-188 days & $\begin{array}{l}\text { N-3: } \uparrow \text { SOD, } \\
\text { glutathione } \\
\text { reductase and } \\
\text { plasma antioxidant } \\
\text { status; } \uparrow \text { QOL }\end{array}$ & $\begin{array}{c}2015 \\
{[16]}\end{array}$ \\
\hline $\begin{array}{l}\text { Gastric } \\
\text { (I-IV) }\end{array}$ & $\begin{array}{l}34(17 / 17) \\
15 \mathrm{~F} / 19 \mathrm{M}\end{array}$ & $\begin{array}{c}\text { Age }=71.2 \pm \\
9.8 / 67.5 \pm 11.2\end{array}$ & Cisplatin & $\begin{array}{c}1.25 \mathrm{~g}=0.92 \mathrm{~g} \\
\mathrm{EPA} / 0.32 \mathrm{~g} \\
\text { DHA } \\
\text { CNT = Placebo }\end{array}$ & 9 weeks & $\begin{array}{c}\text { N-3: } \downarrow \text { gene } \\
\text { expression of } \\
\text { MMP-1 and } \\
\text { MMP-9 compared } \\
\text { to control }\end{array}$ & 2019 [30] \\
\hline
\end{tabular}


Table 1. Cont.

\begin{tabular}{|c|c|c|c|c|c|c|c|}
\hline $\begin{array}{l}\text { Cancer Type } \\
\text { (Stage) }\end{array}$ & $\begin{array}{c}\mathbf{N} \\
\text { (Int/CNT) } \\
\text { Female/Male }\end{array}$ & $\begin{array}{c}\text { Age, Body } \\
\text { Weight and } \\
\text { BMI } \\
\text { (Int/CNT) }\end{array}$ & Chemotherapy & $\begin{array}{c}\mathrm{N}-3(\mathrm{~g} \\
\text { Total/Day = } \\
\text { EPA/DHA) } \\
\text { CNT }\end{array}$ & $\begin{array}{l}\text { Treatment } \\
\text { Duration }\end{array}$ & $\begin{array}{l}\text { Experimental } \\
\text { Findings }\end{array}$ & Ref \\
\hline Gastrointestinal & $\begin{array}{l}38(19 / 19) \\
16 \mathrm{~F} / 22 \mathrm{M}\end{array}$ & $\begin{array}{c}\text { Age }=53.8 \pm \\
2.4 / 54.9 \pm 3.2 \\
\text { Body weight }= \\
65.8 \pm 3.6 / 69.5 \\
\quad \pm 3.6\end{array}$ & $\begin{array}{l}\text { Fluorouracil } \\
\text { and } \\
\text { Leucovorin }\end{array}$ & $\begin{array}{c}0.70 \mathrm{~g}=0.30 \mathrm{~g} \\
\mathrm{EPA} / 0.40 \mathrm{~g} \\
\mathrm{DHA} \\
\mathrm{CNT}=\mathrm{N} / \mathrm{A}\end{array}$ & 8 weeks & $\begin{array}{c}\mathrm{N}-3: \uparrow \text { in } \mathrm{EPA} \text { and } \\
\mathrm{DHA} \text { in PBMCs, } \uparrow \\
\text { in phagocytosis, } \\
\text { superoxide anion } \\
\text { production and } \\
\mathrm{H}_{2} \mathrm{O}_{2} \text { productions, } \\
\uparrow \text { weight, } \\
\text { improved } \\
\text { neutrophil function } \\
\text { during } \\
\text { chemotherapy } \\
\text { Control: } \downarrow \text { weight }\end{array}$ & $\begin{array}{l}2011 \\
{[20]}\end{array}$ \\
\hline Gastrointestinal & $\begin{array}{l}51(26 / 25) \\
24 \mathrm{~F} / 27 \mathrm{M}\end{array}$ & $\begin{array}{c}\text { Age }=58 \\
(46-63) / 51 \\
(41-60) \\
\text { BMI }=26.5 \pm \\
4.6 / 25.6 \pm 4.2 \\
\text { weight loss }= \\
7.2-11.3 \% \text { in } 6 \\
\text { months prior } \\
\text { to study entry }\end{array}$ & $\begin{array}{l}\text { Capecitabine + } \\
\text { Oxaliplatin; } \\
\text { Fluorouracil + } \\
\text { Oxaliplatin; } \\
\text { Fluorouracil + } \\
\text { Leucovorin; } \\
\text { other }\end{array}$ & $\begin{array}{c}1.55 \mathrm{~g}=1.0 \mathrm{~g} \\
\mathrm{EPA} / 0.55 \mathrm{~g} \\
\text { DHA } \\
\text { CNT = olive oil } \\
2 \mathrm{~g}\end{array}$ & 9 weeks & $\begin{array}{l}\text { N-3: } \downarrow \text { in severe } \\
\text { diarrhea compared } \\
\text { to control and } \\
\text { better performance } \\
\text { status score }\end{array}$ & $\begin{array}{l}2019 \\
{[26]}\end{array}$ \\
\hline $\begin{array}{l}\text { Gastrointestinal } \\
\text { (II/III) }\end{array}$ & $\begin{array}{c}81 \\
35 \mathrm{~F} / 46 \mathrm{M}\end{array}$ & $\begin{array}{c}\text { Age }=56.8 \pm \\
10.6 / 59.9 \pm 8.8 \\
\text { Body weight }= \\
68.4 \pm 9.8 / 68.8 \\
\pm 12.0 \\
\text { BMI }=24.3 \pm \\
2.9 / 25.4 \pm 3.6\end{array}$ & Not stated & $\begin{array}{c}0.61 \mathrm{~g}=0.11 \mathrm{~g} \\
\text { EPA } / 0.50 \mathrm{~g} \\
\text { DHA }\end{array}$ & 8 weeks & $\begin{array}{l}\text { N-3: Maintained } \\
\text { weight compared } \\
\text { to control; } \downarrow \text { CRP } \\
\text { compared to } \\
\text { baseline; NS } \\
\text { decrease in TNF } \alpha \\
\text { and IL-6 compared } \\
\text { to baseline; in } \\
\text { combination with } \\
\text { vitamin D } \downarrow \text { CRP, } \\
\text { TNF } \alpha \text { and IL-6 } \\
\text { compared to } \\
\text { baseline } \\
\text { N-3: } \uparrow \text { global }\end{array}$ & 2019 [27] \\
\hline Colorectal & 140 & $\begin{array}{c}\text { Body weight }= \\
54.2 \pm \\
11.7 / 57.4 \pm \\
10.9 \\
\text { BMI }=21.8 \pm \\
4.1 / 23.0 \pm 4.3\end{array}$ & $\begin{array}{l}\text { Capecitabine + } \\
\text { Oxaliplatin }\end{array}$ & $\begin{array}{l}1.40 \mathrm{~g} \mathrm{EPA}+ \\
\text { DHA }\end{array}$ & 8 weeks & $\begin{array}{l}\text { health status, } \downarrow \\
\text { fatigue, nausea, } \\
\text { pain, } \downarrow \text { IL-6 } \\
\text { compared to } \\
\text { baseline and } \\
\text { control; NC TNF } \alpha \\
\text { or CRP }\end{array}$ & $\begin{array}{l}2018 \\
{[45]}\end{array}$ \\
\hline $\begin{array}{l}\text { Colorectal } \\
\text { (III and IV) }\end{array}$ & $\begin{array}{l}11(6 / 5) \\
5 \mathrm{~F} / 6 \mathrm{M}\end{array}$ & $\begin{array}{c}\text { Age }=53.6 \pm \\
12.9 / 55.2 \pm 7.7 \\
\text { Body weight }= \\
72.3 \pm \\
12.3 / 68.1 \pm \\
12.1 \\
\text { BMI }=28.6 \pm \\
6.3 / 26.4 \pm 3.7 \\
\text { Age }=50.1 \pm\end{array}$ & $\begin{array}{l}\text { Xeloda, } \\
\text { Oxaliplatin, } \\
\text { Fluorouracil } \\
\text { and/or } \\
\text { Leucovorin }\end{array}$ & $\begin{array}{c}0.60 \mathrm{~g}=0.36 \mathrm{~g} \\
\mathrm{EPA} / 0.24 \mathrm{~g} \\
\mathrm{DHA} \\
\mathrm{CNT}=\mathrm{N} / \mathrm{A}\end{array}$ & 9 weeks & $\begin{array}{l}\text { N-3: Improved } \\
\text { CRP, CRP/albumin } \\
\text { and potentially } \\
\text { prevented weight } \\
\text { loss }\end{array}$ & $\begin{array}{l}2013 \\
{[32]}\end{array}$ \\
\hline $\begin{array}{l}\text { Colorectal and } \\
\text { Rectal }\end{array}$ & $\begin{array}{l}23(11 / 12) \\
6 \mathrm{~F} / 17 \mathrm{M}\end{array}$ & $\begin{array}{c}8.2 / 54.3 \pm 9.3 \\
\text { Body weight }= \\
73 \pm 16.8 / 66.8 \\
\pm 11.6 \\
\text { BMI }=27.3 \pm \\
6.1 / 25.0 \pm 3.4\end{array}$ & $\begin{array}{l}\text { Type not } \\
\text { specified }\end{array}$ & $\begin{array}{c}0.60 \mathrm{~g}=0.36 \mathrm{~g} \\
\mathrm{EPA} / 0.24 \mathrm{~g} \\
\mathrm{DHA} \\
\mathrm{CNT}=\mathrm{N} / \mathrm{A}\end{array}$ & 9 weeks & $\begin{array}{c}\mathrm{N}-3: \downarrow \\
\text { CRP/albumin ratio }\end{array}$ & $\begin{array}{l}2012 \\
{[33]}\end{array}$ \\
\hline $\begin{array}{l}\text { Colorectal } \\
\text { (II-IV) }\end{array}$ & $\begin{array}{c}30 \\
(17 / 13) 10 \\
\mathrm{~F} / 20 \mathrm{M}\end{array}$ & $\begin{array}{c}\text { Age }=52.1 \pm \\
7.6 / 53.1 \pm 10.2\end{array}$ & $\begin{array}{l}\text { Chemotherapy } \\
\text { type not stated }\end{array}$ & $\begin{array}{c}0.60 \mathrm{~g}=0.36 \mathrm{~g} \\
\mathrm{EPA} / 0.24 \mathrm{~g} \\
\mathrm{DHA} \\
\mathrm{CNT}=\mathrm{N} / \mathrm{A}\end{array}$ & 9 weeks & $\begin{array}{c}\mathrm{N}-3: \uparrow \text { time to } \\
\text { progression }(20 \mathrm{vs.} \\
11 \text { months); } \downarrow \\
\text { carcinoembryonic } \\
\text { antigen }\end{array}$ & $\begin{array}{l}2016 \\
{[34]}\end{array}$ \\
\hline
\end{tabular}


Table 1. Cont.

\begin{tabular}{|c|c|c|c|c|c|c|c|}
\hline $\begin{array}{c}\text { Cancer Type } \\
\text { (Stage) }\end{array}$ & $\begin{array}{c}\text { N } \\
(\text { Int/CNT) } \\
\text { Female/Male }\end{array}$ & $\begin{array}{l}\text { Age, Body } \\
\text { Weight and } \\
\text { BMI } \\
\text { (Int/CNT) }\end{array}$ & Chemotherapy & $\begin{array}{c}\mathrm{N}-3(\mathrm{~g} \\
\text { Total/Day }= \\
\text { EPA/DHA) } \\
\text { CNT }\end{array}$ & $\begin{array}{l}\text { Treatment } \\
\text { Duration }\end{array}$ & $\begin{array}{l}\text { Experimental } \\
\text { Findings }\end{array}$ & Ref \\
\hline $\begin{array}{c}\text { Leukemia } \\
\text { Lymphoma }\end{array}$ & $\begin{array}{c}22(9 / 13) \\
10 \mathrm{~F} / 12 \mathrm{M}\end{array}$ & $\begin{array}{c}\text { Age }= \\
43.8 / 53.8 \\
\text { Body weight }= \\
68.1 \pm \\
10.3 / 72.4 \pm \\
11.6 \\
\text { BMI }=24.6 \pm \\
4.1 / 25.7 \pm 4.0\end{array}$ & $\begin{array}{l}\text { Type not } \\
\text { specified }\end{array}$ & $\begin{array}{c}0.61 \mathrm{~g}=0.37 \mathrm{~g} \\
\mathrm{EPA} / 0.24 \mathrm{~g} \\
\mathrm{DHA} \\
\mathrm{CNT}=\mathrm{N} / \mathrm{A}\end{array}$ & 9 weeks & $\begin{array}{c}\text { N-3: } \downarrow \\
\text { CRP/albumin ratio } \\
\text { from high to low; } \uparrow \\
\text { overall long-term } \\
\text { survival (at } 465 \\
\text { days) compared to } \\
\text { control } \\
\text { Control: } \\
\downarrow \text { CRP / albumin } \\
\text { ratio from high to } \\
\text { medium }\end{array}$ & $\begin{array}{l}2017 \\
{[48]}\end{array}$ \\
\hline $\begin{array}{l}\text { NSCLC } \\
\text { (III or IV) }\end{array}$ & $\begin{array}{l}46(31 / 15) \\
22 \mathrm{~F} / 24 \mathrm{M}\end{array}$ & $\begin{array}{l}\text { Age }=64 \pm \\
1.7 / 63 \pm 2.1\end{array}$ & $\begin{array}{l}\text { Carboplatin } \\
\text { and } \\
\text { Vinorelbine or } \\
\text { Carboplatin } \\
\text { and } \\
\text { Gemcitabine }\end{array}$ & $\begin{array}{c}2.4-2.7 \mathrm{~g}=2.2 \\
\mathrm{~g} \mathrm{EPA}+ \\
0.24-0.50 \mathrm{~g} \\
\text { DHA } \\
\mathrm{CNT}=\mathrm{SOC}\end{array}$ & 6 weeks & $\begin{array}{c}\text { N-3: } \uparrow \\
\text { chemotherapy } \\
\text { response rate, } \uparrow \\
\text { clinical benefit; } \uparrow \\
\text { 1-year survival } \\
\text { (trend) }\end{array}$ & 2011 [49] \\
\hline $\begin{array}{c}\text { Lung } \\
\text { (Advanced) }\end{array}$ & $\begin{array}{l}27(13 / 14) \\
8 \mathrm{~F} / 19 \mathrm{M}\end{array}$ & $\begin{array}{c}\text { Age }=55.6 \pm \\
7.4 / 60.6 \pm 7.4 \\
\text { Body weight }= \\
75.1 \pm \\
16.1 / 68.0 \pm \\
12.8 \\
\text { BMI }=26.2 \pm \\
7.0 / 25.2 \pm 3.9\end{array}$ & $\begin{array}{l}\text { Gemcitabine, } \\
\text { Cisplatin }\end{array}$ & $\begin{array}{c}3.4 \mathrm{~g}=2.0 \mathrm{~g} \\
\mathrm{EPA} / 1.4 \mathrm{~g} \\
\text { DHA } \\
\mathrm{CNT}=\text { olive oil }\end{array}$ & 66 days & $\begin{array}{c}\text { N-3: } \uparrow \text { in EPA }+ \\
\text { DHA in plasma, } \uparrow \\
\text { in EPA in RBC; } \downarrow \\
\text { IL-6, PGE2 and } \uparrow \\
\text { Body weight; } \downarrow \\
\text { inflammatory } \\
\text { indexes and } \\
\text { oxidative status; } \\
\text { Control: } \uparrow \text { CRP, } \\
\text { IL-6, TNF and ROS }\end{array}$ & $\begin{array}{c}2012 \\
{[50]}\end{array}$ \\
\hline $\begin{array}{c}\text { NSCLC } \\
\text { (Advanced) }\end{array}$ & $\begin{array}{c}137(77 / 60) 61 \\
\mathrm{~F} / 76 \mathrm{M}\end{array}$ & $\begin{array}{c}\text { Age }=63.8 \pm \\
6.4 / 62.9 \pm 7.1 \\
\text { Body weight }= \\
67.2 \pm \\
11.5 / 70.1 \pm \\
12.3 \\
\text { BMI }=23.5 \pm \\
2.1 / 23.9 \pm 2.4\end{array}$ & $\begin{array}{c}\text { Cisplatin, } \\
\pm \mathrm{TXT}, \pm \\
\text { Bevacizumab }\end{array}$ & $\begin{array}{c}0.71 \mathrm{~g}=0.5 \mathrm{~g} \\
\mathrm{EPA} / 0.20 \mathrm{~g} \\
\mathrm{DHA} \\
\mathrm{CNT}=\mathrm{N} / \mathrm{A}\end{array}$ & 6 weeks & $\begin{array}{c}\text { N-3 group } \downarrow \text { CRP, } \\
\text { IL-6 and PGE2; NC } \\
\text { in QOL or } \\
\text { nutritional status }\end{array}$ & $\begin{array}{c}2018 \\
{[51]}\end{array}$ \\
\hline $\begin{array}{l}\text { Multiple } \\
\text { Myeloma }\end{array}$ & $\begin{array}{c}18 \\
8 \mathrm{~F} / 11 \mathrm{M}\end{array}$ & $\begin{array}{c}\text { Age }=69 \\
(57-76)\end{array}$ & $\begin{array}{c}\text { Bortezomib + } \\
\text { Thalidomide + } \\
\text { Dexametha- } \\
\text { sone (84 days) } \\
\text { or Bortezomib } \\
+ \text { Melphalan + } \\
\text { Prednisone }\end{array}$ & $\begin{array}{c}2 \mathrm{~g}=1.2 \mathrm{~g} \text { ALA } \\
+0.80 \mathrm{~g} \text { DHA } \\
\mathrm{CNT}=\mathrm{N} / \mathrm{A}\end{array}$ & 6 months & $\begin{array}{c}\text { N-3: } \downarrow \text { in onset or } \\
\text { worsening of } \\
\text { neuropathic pain, } \downarrow \\
\text { in chemotherapy } \\
\text { interruptions }\end{array}$ & 2018 [56] \\
\hline
\end{tabular}

Abbreviations used: ALA, alpha linolenic acid; BMI, body mass index; CI, confidence interval; CNT, control; CRP, C-reactive protein; DFS, disease-free survival; DHA, docosahexaenoic acid; EPA, eicosapentaenoic acid; F, female; g, gram $(\mathrm{s}) ; \mathrm{H}_{2} \mathrm{O}_{2}$, hydrogen peroxide; HR, hazards ratio; IL, interleukin; Int, intervention group; M, male; MMP, matrix metalloproteinase; N, number; N/A, not applicable; NC, no change, NS, non-significant; NSCLC, non-small-cell lung cancer; OS, overall survival; PBMC, peripheral blood mononuclear cell, PGE2, prostaglandin E2; QOL, quality of life; RBC, red blood cell; Ref, reference; SOC, standard of care; SOD, super oxide dismutase; TNF $\alpha$, tumor necrosis factor; VEGF, vascular endothelial growth factor. 
Table 2. Randomized controlled trials providing N-3 supplementation without chemotherapy.

\begin{tabular}{|c|c|c|c|c|c|c|c|}
\hline $\begin{array}{c}\text { Cancer Type } \\
\text { (Stage) }\end{array}$ & $\begin{array}{c}\mathrm{N} \\
(\text { Int/CNT) } \\
\text { Female/Male }\end{array}$ & $\begin{array}{c}\text { Age, Body } \\
\text { Weight and } \\
\text { BMI (Int/CNT) }\end{array}$ & Chemotherapy & $\begin{array}{c}\text { N-3 (g EPA + } \\
\text { DHA/Day) } \\
\text { CNT }\end{array}$ & $\begin{array}{l}\text { Treatment } \\
\text { Duration }\end{array}$ & $\begin{array}{l}\text { Experimental } \\
\text { Findings }\end{array}$ & Ref \\
\hline $\begin{array}{l}\text { Breast } \\
\text { (I-III) }\end{array}$ & $\begin{array}{c}37 \mathrm{~F} \\
(18 / 19)\end{array}$ & $\begin{array}{c}\text { Age }=48.6+ \\
9.0 / 53.4+7.5 \\
\text { BMI }=43 \% \\
\text { overweight, } 30 \% \\
\text { obese }\end{array}$ & $\begin{array}{c}\text { No } \\
\text { chemotherapy- } \\
\text { N-3 } \\
\text { supplementation } \\
\text { prior to } \\
\text { treatment } \\
\text { Previous } \\
\text { chemotherapy }\end{array}$ & $\begin{array}{c}0.94 \mathrm{~g} \mathrm{EPA}+0.78 \\
\text { g DHA } \\
\mathrm{CNT}=2 \mathrm{~g} \\
\text { mineral oil }\end{array}$ & 30 days & $\begin{array}{c}\text { N-3: NC CD4+, } \\
\text { CD8+, PGE2, IL-6 } \\
\text { Control: } \downarrow \text { CD4+, NC } \\
\text { PGE2, IL-6, } \uparrow \text { hsCRP }\end{array}$ & 2017 [12] \\
\hline $\begin{array}{l}\text { Breast } \\
\text { (I-III) }\end{array}$ & 45 & $\begin{array}{c}\text { Age }=57.3 \\
(40-81) \\
\text { BMI }=28.9 \\
(19.3-38.3)\end{array}$ & $\begin{array}{c}(69.9 \%), \text { previous } \\
\text { radiotherapy } \\
(87 \%) \text {; currently } \\
\text { on aromatase } \\
\text { inhibitors } 67.3 \% \\
\text { or Tamoxifen } \\
32.6 \%\end{array}$ & $\begin{array}{c}1.38 \mathrm{~g} \mathrm{~N}-3 \\
\mathrm{CNT}=\mathrm{N} / \mathrm{A}\end{array}$ & 30 days & $\begin{array}{c}\text { N-3: } \downarrow \text { from baseline } \\
\text { at day } 30 \text { and day } 60 \\
\text { of CRP; } 21.5 \% \\
\text { decrease in pain scale; } \\
\downarrow \text { in IFN } \gamma \text { at day } 30\end{array}$ & 2019 [13] \\
\hline $\begin{array}{c}\text { Breast, } \\
\text { gastrointestinal, } \\
\text { lung, liver, } \\
\text { pancreas } \\
\text { (all } \\
\text { metastasized) }\end{array}$ & $\begin{array}{c}64(60 \\
\text { completed }) \\
24 \mathrm{~F} / 36 \mathrm{M}\end{array}$ & $\begin{array}{l}\text { Age }=60 \pm 5(\mathrm{~F}) \\
57 \pm 4(\mathrm{M}) / 58 \pm \\
4(\mathrm{~F}), 56 \pm 3(\mathrm{M})\end{array}$ & $\begin{array}{l}\text { Previous surgery } \\
n=38, \text { Previous } \\
\text { chemotherapy } n \\
=26, \text { previous } \\
\text { radiotherapy } n= \\
6, \text { none }=10\end{array}$ & $\begin{array}{c}3.1 \mathrm{~g} \mathrm{EPA}+2.1 \mathrm{~g} \\
\mathrm{DHA} \\
\mathrm{CNT}=\text { sugar } \\
\text { tablets }\end{array}$ & until death & $\begin{array}{l}\text { Both groups: } \uparrow \text { in } \\
\text { survival in } \\
\text { well-nourished vs. } \\
\text { malnourished } \\
\text { N-3: } \uparrow \text { in survival, } \uparrow \\
\text { CD4/CD8 }\end{array}$ & 1998 [14] \\
\hline $\begin{array}{l}\text { Colorectal } \\
\text { (local and } \\
\text { advanced) }\end{array}$ & 30 & Age $=63 \pm 2.3$ & Surgery & $\begin{array}{c}\text { Group } 1 \\
\text { (localized): } 1.2 \mathrm{~g} \\
\text { GLA + 1.1 g EPA } \\
\text { + 0.16 g DHA } \\
\text { Group } 2 \\
\text { (advanced): } \\
\text { T0-15 1.2 g GLA } \\
\text { + } 1.1 \mathrm{~g} \text { EPA + } \\
0.16 \text { g DHA, } \\
\text { T16-30: } 1.8 \text { g } \\
\text { GLA + 1.6 g EPA } \\
\text { + 0.24 g DHA; } \\
\text { Months 2-6: } 2.3 \\
\text { g GLA + 2.1 g } \\
\text { EPA + 0.32 g } \\
\text { DHA } \\
\text { Group 3: CNT (6 } \\
\text { months) }\end{array}$ & $\begin{array}{l}\text { Group 1: until } \\
\text { surgery } \\
\text { Group } 2 \text { and 3: } 6 \\
\text { months }\end{array}$ & $\begin{array}{c}\text { Group } 1: \text { NC in } \\
\text { immune parameters; } \\
\text { Group } 2=\downarrow \text { IL1 } \beta 3,4 \text {, } \\
5 \text { and } 6 \text { months; } \downarrow \\
\text { IL- } 4 \text { at } 2,3,4,5 \text { and } 6 ; \\
\downarrow \text { IL- } 6 \text { at } 6 \text { months; } \downarrow \\
\text { TNF } \alpha \text { at } 2,4,5 \text { and } 6 ; \\
\downarrow \text { IFN } \gamma \text { by month } 4 \\
\text { Group } 3 \text { : NC }\end{array}$ & 1994 [35] \\
\hline $\begin{array}{l}\text { Colorectal } \\
\text { (Dukes A-D) } \\
\text { All with liver } \\
\text { metastases }\end{array}$ & $\begin{array}{c}88 \\
43(17 \mathrm{~F} / 26 \\
\mathrm{M}) / 45(10 \mathrm{~F} / 35 \\
\mathrm{M})\end{array}$ & $\begin{array}{c}\text { Age }=71 \\
(35-87) / 68 \\
(44-82)\end{array}$ & $\begin{array}{c}\text { Previous } \\
\text { chemotherapy }\end{array}$ & $\begin{array}{c}2 \mathrm{~g} \text { EPA } \\
\mathrm{CNT}=2 \mathrm{~g} \mathrm{MCT}\end{array}$ & 12-65 days & $\begin{array}{c}\text { N-3: } \uparrow \text { EPA in tumor } \\
\text { tissue, NC in Ki67, } \uparrow \\
\text { OS at } 18 \text { months } \\
\text { (trend) }\end{array}$ & 2014 [36] \\
\hline $\begin{array}{l}\text { Advanced lung } \\
\text { (III-IV) }\end{array}$ & $\begin{array}{l}22(10 / 12) \\
5 \mathrm{~F} / 17 \mathrm{M}\end{array}$ & $\begin{array}{c}\text { Age }=64 \\
(44-90) / 61 \\
(44-83) \\
\text { Body weight }= \\
60.1 \pm 8.2 / 62.8 \\
\pm 9.7 \\
\text { BMI }=24 \pm \\
6.2 / 25.8 \pm 4.4\end{array}$ & $\mathrm{~N} / \mathrm{A}$ & $\begin{array}{c}0.36 \mathrm{~g} \mathrm{EPA}+0.24 \\
\text { g DHA + } \\
\text { celecoxib } \\
\mathrm{CNT}=0.36 \mathrm{~g} \\
\mathrm{EPA}+0.24 \mathrm{~g} \\
\mathrm{DHA}\end{array}$ & 6 weeks & $\begin{array}{c}\text { N-3 + celecoxib: } \downarrow \\
\text { CRP; } \uparrow \text { body weight } \\
\text { and hand grip scores } \\
\text { improved } \\
\text { N-3 alone: } \downarrow \text { CRP }\end{array}$ & $\begin{array}{l}2007 \\
{[52]}\end{array}$ \\
\hline $\begin{array}{l}\text { Pancreatic } \\
\text { (II-IV) }\end{array}$ & $\begin{array}{c}26 \\
14 \mathrm{~F} / 12 \mathrm{M}\end{array}$ & $\begin{array}{c}\text { Age }=56(39-75) \\
\text { Body weight }= \\
66.8(56.0-75.1) \\
\text { BMI = 23.2 } \\
(21.1-27.4)\end{array}$ & $\mathrm{N} / \mathrm{A}$ & $\begin{array}{c}\text { EPA only week } 1: \\
1 \mathrm{~g} \text { week } 2: 2 \mathrm{~g} \\
\text { week 3: } 4 \mathrm{~g} \text { week } \\
\text { 4-12: } 6 \mathrm{~g} \\
\mathrm{CNT}=\mathrm{N} / \mathrm{A}\end{array}$ & $\begin{array}{c}12 \text { weeks until } \\
\text { death }\end{array}$ & $\begin{array}{c}\text { Body weight } \\
\text { stabilized and began } \\
\text { to increase by week } 4 ; \\
\text { CRP stabilized or was } \\
\text { slightly reduced in } \\
\text { patients who had } \uparrow \\
\text { CRP at beginning; } \\
\text { median survival = } 6.8 \\
\text { months }\end{array}$ & $\begin{array}{l}2000 \\
{[57]}\end{array}$ \\
\hline Pancreatic & $\begin{array}{l}33(18 / 15) \\
17 \mathrm{~F} / 16 \mathrm{M}\end{array}$ & $\begin{array}{c}\text { Age }=70.3 \pm \\
8.2 / 71.3 \pm 7.5 \\
\text { Body weight }= \\
62.9 \pm 6.5 / 71.4 \\
\pm 15.3 \\
\text { BMI }=21.3 \pm \\
1.7 / 23.7 \pm 4.1\end{array}$ & $\begin{array}{c}24 \text { patients } \\
\text { received } \\
\text { chemotherapy, } 2 \\
\text { received } \\
\text { radiotherapy } \\
\text { (not all curative, } \\
\text { most palliative) }\end{array}$ & $\begin{array}{c}\text { Group 1: } 0.10 \mathrm{~g} \\
\text { EPA + 0.20 g } \\
\text { DHA; } \\
\text { Group 2: } 0.13 \mathrm{~g} \\
\text { EPA + 0.18 g } \\
\text { DHA } \\
\text { CNT }=\text { N } / A\end{array}$ & 6 weeks & $\uparrow$ in HDL in Group 1 & $\begin{array}{l}2017 \\
{[58]}\end{array}$ \\
\hline
\end{tabular}

Abbreviations used: BMI, body mass index; CD, cluster of differentiation; CNT, control; CRP, C-reactive protein; DHA, docosahexaenoic acid; EPA, eicosapentaenoic acid; F, female; g, gram(s); GLA, gamma linolenic acid; HDL, high-density lipoprotein; hsCRP, high sensitivity $\mathrm{CRP}$; IFN $\gamma$, interferon gamma; IL, interleukin; Int, intervention group; $\mathrm{M}$, male; MCT, medium chain triglycerides; N, number; N/A, not applicable; NC, no change, OS, overall survival; PGE2, prostaglandin E2; Ref, reference; SOC, standard of care; TNF $\alpha$, tumor necrosis factor. 
Table 3. Randomized controlled trials providing oral N-3 supplementation.

\begin{tabular}{|c|c|c|c|c|c|c|c|}
\hline $\begin{array}{c}\text { Cancer Type } \\
\text { (Stage) }\end{array}$ & $\begin{array}{c}\mathbf{N} \\
\text { (Int/CNT) } \\
\text { Female/Male }\end{array}$ & $\begin{array}{c}\text { Age } \\
\text { Body Weight } \\
\text { and } \\
\text { BMI } \\
\text { (Int/CNT) }\end{array}$ & Chemotherapy & $\begin{array}{c}\text { N-3 (g EPA + } \\
\text { DHA/Day) } \\
\text { CNT }\end{array}$ & $\begin{array}{l}\text { Treatment } \\
\text { Duration }\end{array}$ & $\begin{array}{l}\text { Experimental } \\
\text { Findings }\end{array}$ & Ref \\
\hline $\begin{array}{l}\text { Lung, Head } \\
\text { and Neck, } \\
\text { Gynecologic, } \\
\text { Breast, } \\
\text { Prostate, } \\
\text { Urinary Tract, } \\
\text { Esophagus } \\
\text { (I-IV) }\end{array}$ & $\begin{array}{c}38 \text { 20/18 } \\
14 \mathrm{~F} / 24 \mathrm{M}\end{array}$ & $\begin{array}{c}\text { Age }=62.7 \pm \\
11.0 \\
\text { Body weight }= \\
70.8 \pm 12.6 \\
\text { BMI }=24.8 \pm \\
3.5\end{array}$ & Radiotherapy & $\begin{array}{c}2 \times 326 \mathrm{kcal}: \\
2.4 \mathrm{~g} \mathrm{EPA}+1.2 \\
\mathrm{~g} \text { DHA }+40 \mathrm{~g} \\
\text { protein } \\
\mathrm{CNT}=\mathrm{N} / \mathrm{A}\end{array}$ & 7 days & $\begin{array}{c}\text { N-3: } \downarrow \text { serum PGE2 } \\
\text { Control: } \uparrow \text { serum } \\
\text { PGE2 } \\
\text { No differences in } \\
\text { cytokine } \\
\text { production }\end{array}$ & $\begin{array}{l}2013 \\
{[15]}\end{array}$ \\
\hline $\begin{array}{l}\text { Stomach, } \\
\text { Colon, Lung, } \\
\text { Pancreas, } \\
\text { Other }\end{array}$ & 40 & $\begin{array}{c}\text { Age }=61.3 \pm \\
12.1 / 63.6 \pm \\
11.4 \\
\text { BMI }=20.9 \pm \\
3.7 / 22.2 \pm 3.8\end{array}$ & $\begin{array}{l}\text { Chemotherapy } \\
\pm \text { radiation or } \\
\text { no treatment }\end{array}$ & $\begin{array}{c}600 \text { kcal: } 1.5 \mathrm{~g} \\
\text { EPA }+19.5 \% \\
\text { protein } \\
\text { CNT }= \\
\text { isocaloric } \\
\text { supplement }\end{array}$ & 1 month & $\begin{array}{c}\text { Both groups: } \uparrow \\
\text { SF36 } \\
\text { N-3: } \downarrow \text { in IFN } \gamma \\
\text { Control: } \uparrow \text { in IFN } \gamma\end{array}$ & $\begin{array}{l}2011 \\
{[21]}\end{array}$ \\
\hline $\begin{array}{l}\text { Head and } \\
\text { Neck } \\
\text { (I-IV) }\end{array}$ & $\begin{array}{l}27(13 / 14) \\
11 \mathrm{~F} / 16 \mathrm{M}\end{array}$ & $\begin{array}{c}\text { Age }=61.5(45- \\
77) / 66.1 \\
(47-76) \\
\text { BMI }=\text { NS but } \\
\text { cachexic } \\
\text { Age }=60 \pm\end{array}$ & Surgery & $\begin{array}{c}600 \mathrm{kcal}: 2.1 \mathrm{~g} \\
\mathrm{EPA}+32 \mathrm{~g} \\
\text { protein } \\
\mathrm{CNT}=\mathrm{N} / \mathrm{A}\end{array}$ & 4 weeks & $\begin{array}{l}\text { No differences } \\
\text { between groups or } \\
\text { from baseline }\end{array}$ & $\begin{array}{l}2018 \\
{[17]}\end{array}$ \\
\hline $\begin{array}{l}\text { Head and } \\
\text { Neck } \\
\text { (I-IV) }\end{array}$ & $\begin{array}{c}64 \\
29 \mathrm{~F} / 35 \mathrm{M}\end{array}$ & $\begin{array}{c}\text { Body weight }= \\
58.8 \pm 1.4 / 61.1 \\
\pm 11.5 \\
\text { BMI }=22.6 \pm \\
4.6 / 24 \pm 4.2 \\
\text { weight loss }= \\
\sim 9 \mathrm{~kg} \text { in } 3 \\
\text { months before } \\
\text { entry }\end{array}$ & $\begin{array}{l}\text { Surgery, } \\
\text { radiotherapy, } \\
\text { chemotherapy, } \\
\text { or combination }\end{array}$ & $\begin{array}{c}600 \mathrm{kcal}: 2 \mathrm{~g} \\
\mathrm{EPA}+40 \mathrm{~g} \\
\text { protein } \\
\mathrm{CNT}= \\
\text { isocaloric } \\
\text { supplement }\end{array}$ & 6 weeks & $\begin{array}{c}\text { N-3: weight } \\
\text { maintenance, } \downarrow \text { CRP, } \\
\text { TNF } \alpha \text { and IFN } \gamma \\
\text { Control: weight } \\
\text { loss }(2.0 \pm 3.7 \mathrm{lbs}), \\
\downarrow \mathrm{CRP}, \uparrow \mathrm{TNF} \alpha \text { and } \\
\text { IFN } \gamma\end{array}$ & $\begin{array}{l}2018 \\
{[18]}\end{array}$ \\
\hline $\begin{array}{l}\text { Colorectal } \\
\text { (IV) }\end{array}$ & $\begin{array}{c}23 \\
8 \mathrm{~F} / 13 \mathrm{M}\end{array}$ & $\begin{array}{c}\text { Age }=61 \pm \\
11.6 \\
\text { Body weight }= \\
75.9 \pm 17.0 \\
\text { BMI }=28 \pm 6.4\end{array}$ & $\begin{array}{l}\text { Chemotherapy } \\
17 \text { with } \\
\text { previous } \\
\text { chemotherapy }\end{array}$ & $\begin{array}{c}600 \text { kcal: } 2.2 \mathrm{~g} \\
\mathrm{EPA}+0.92 \mathrm{~g} \\
\mathrm{DHA}+32 \mathrm{~g} \\
\text { protein } \\
\mathrm{CNT}=\mathrm{N} / \mathrm{A}\end{array}$ & 9 weeks & $\begin{array}{c}\text { N-3: } \downarrow \text { in GM-CSF, } \\
\uparrow \text { RANTES, CRP } \\
\text { (week 3) } \\
\uparrow \text { in GM-CSF and } \\
\text { NC CRP } \\
\text { (compared to } \\
\text { baseline; week 9), } \\
\text { Correlations } \\
\text { between baseline } \\
\text { IL-10 and survival, } \\
\text { IL-6 and survival, } \\
\text { IL-6 and CRP }\end{array}$ & $\begin{array}{l}2007 \\
{[41]}\end{array}$ \\
\hline $\begin{array}{l}\text { Colorectal } \\
\text { (IV) }\end{array}$ & $\begin{array}{l}13(5 / 6) \\
4 \mathrm{~F} / 9 \mathrm{M}\end{array}$ & $\begin{array}{c}\text { Age }=61.5 \pm \\
15.8 / 68.2 \pm \\
15.6 \\
\text { Body weight }= \\
69.9 \pm \\
15.9 / 72.2 \pm \\
11.7 \mathrm{BMI}=25.8 \\
\pm 4.3 / 26 \pm 3.3 \\
\text { Age }=58\end{array}$ & $\begin{array}{l}\text { Fluorouracil + } \\
\text { oxaliplatin + } \\
\text { folinic acid or } \\
\text { capecitabine }\end{array}$ & $\begin{array}{c}600 \mathrm{kcal}: 2 \mathrm{~g} \\
\mathrm{EPA}+0.9 \mathrm{~g} \\
\mathrm{DHA}+32 \mathrm{~g} \\
\text { protein } \\
\mathrm{CNT}=\mathrm{N} / \mathrm{A}\end{array}$ & 12 weeks & $\begin{array}{c}\text { N-3: } \uparrow \text { weight, NS } \\
\text { improvement in } \\
\text { QOL and appetite, } \\
\text { NS } \downarrow \text { in fatigue and } \\
\text { pain }\end{array}$ & $\begin{array}{l}2010 \\
{[37]}\end{array}$ \\
\hline $\begin{array}{l}\text { Gastric } \\
\text { (I-IV) }\end{array}$ & $\begin{array}{c}68 \\
24 \mathrm{~F} / 44 \mathrm{M}\end{array}$ & $\begin{array}{c}\text { Body weight }= \\
63.5(58.1- \\
69.8) / 66.1 \\
(71.7-75.4) \\
\text { BMI }=24.2 \\
(20.4- \\
26.3) / 22.8 \\
(20.1-28.3)\end{array}$ & Not stated & $\begin{array}{c}600 \mathrm{kcal}: 2 \mathrm{~g} \\
\mathrm{EPA}+1.2 \mathrm{~g} \\
\mathrm{DHA}+24 \mathrm{~g} \\
\text { protein } \\
\mathrm{CNT}= \\
\text { isocaloric } \\
\text { supplement }\end{array}$ & 30 days & $\begin{array}{c}\text { N-3: } \uparrow \text { weight and } \\
\downarrow \text { IL- } 6 \text { compared to } \\
\text { control }\end{array}$ & $\begin{array}{l}2018 \\
{[31]}\end{array}$ \\
\hline
\end{tabular}


Table 3. Cont.

\begin{tabular}{|c|c|c|c|c|c|c|c|}
\hline $\begin{array}{c}\text { Cancer Type } \\
\text { (Stage) }\end{array}$ & $\begin{array}{c}\mathbf{N} \\
\text { (Int/CNT) } \\
\text { Female/Male }\end{array}$ & $\begin{array}{c}\text { Age } \\
\text { Body Weight } \\
\text { and } \\
\text { BMI } \\
\text { (Int/CNT) }\end{array}$ & Chemotherapy & $\begin{array}{c}\text { N-3 (g EPA + } \\
\text { DHA/Day) } \\
\text { CNT }\end{array}$ & $\begin{array}{l}\text { Treatment } \\
\text { Duration }\end{array}$ & $\begin{array}{l}\text { Experimental } \\
\text { Findings }\end{array}$ & Ref \\
\hline Gastrointestinal & $\begin{array}{c}42 \\
15 \mathrm{~F} / 27 \mathrm{M}\end{array}$ & $\begin{array}{c}\text { Age }= \\
68.1 / 66.7 \\
\text { Body weight }= \\
69.1 / 67.8\end{array}$ & Surgery & $\begin{array}{l}10.5 \% \mathrm{n}-3 \text { of } \\
25 \% \text { fat }+5.6 \mathrm{~g} \\
\text { protein in } 100 \\
\mathrm{~mL} \text { (patients } \\
\text { received } 25 \\
\mathrm{kcal} / \mathrm{kg} \text { body } \\
\quad \text { weight) }\end{array}$ & $\begin{array}{c}16 \text { days } \\
\text { postoperative }\end{array}$ & $\begin{array}{c}\text { N-3: NC in } \\
\text { albumin, } \\
\text { transferrin, } \\
\text { prealbumin, PHA; } \\
\uparrow \text { stimulated IFN, } \\
\text { CD3+, CD3+ } \\
\text { HLA-DR, CD4+ } \\
\text { and B } \\
\text { lymphocytesBoth } \\
\text { groups: } \downarrow \text { T } \\
\text { lymphocytes } \\
\text { (preoperative to } \\
\text { postoperative) }\end{array}$ & $\begin{array}{l}1995 \\
{[38]}\end{array}$ \\
\hline $\begin{array}{l}\text { Gastrointestinal } \\
\text { (Advanced) }\end{array}$ & $\begin{array}{c}24 \\
10 \mathrm{~F} / 14 \mathrm{M}\end{array}$ & $\begin{array}{c}\text { Age }=66 \pm \\
9 / 69 \pm 10 \\
\text { Body weight }= \\
56.6(35- \\
101) / 61.8(33- \\
80) \mathrm{BMI}=21.6 \\
\pm 4.1 / 21.1 \pm \\
4.8 \\
\text { All had }>10 \% \\
\text { weight loss in } \\
\text { past } 6 \text { months }\end{array}$ & $\begin{array}{l}\text { Palliative (at } \\
\text { least } 2 \text { rounds } \\
\text { of } \\
\text { chemotherapy } \\
\text { before study } \\
\text { entry) }\end{array}$ & $\begin{array}{c}4.9 \mathrm{~g} \text { EPA and } \\
3.2 \mathrm{~g} \text { DHA } \pm \\
\text { melatonin } \\
\mathrm{CNT}= \\
\text { isocaloric } \\
\text { supplement }\end{array}$ & 4 weeks & $\begin{array}{c}\text { N-3: } 38 \% \text { had } \\
\text { weight } \\
\text { maintenance, No } \\
\text { statistically } \\
\text { significant changes } \\
\text { in cytokines }\end{array}$ & $\begin{array}{l}2005 \\
{[39]}\end{array}$ \\
\hline $\begin{array}{l}\text { Gastrointestinal } \\
\text { (II-IV) }\end{array}$ & $\begin{array}{c}128 \\
38 \mathrm{~F} / 90 \mathrm{M}\end{array}$ & $\begin{array}{c}\text { Age }=72.3 \pm \\
8.4 / 68.9 \pm 10.3 \\
\text { Body weight }= \\
\text { NS but } 5 \% \\
\text { weight loss } \\
\text { before entry }\end{array}$ & $\begin{array}{l}44 \text { adjuvant } \\
\text { chemother- } \\
\text { apy/84 } \\
\text { palliative } \\
\text { chemotherapy }\end{array}$ & $\begin{array}{c}600 \text { kcal } \\
\text { supplement: } \\
2.2 \mathrm{~g} \mathrm{EPA}+ \\
0.92 \mathrm{~g} \mathrm{DHA}+ \\
32 \mathrm{~g} \text { protein } \\
\mathrm{CNT}=\mathrm{N} / \mathrm{A}\end{array}$ & 6 months & $\begin{array}{l}\text { N-3: stable CRP } \\
\text { Control: } \uparrow \text { CRP }\end{array}$ & $\begin{array}{l}2017 \\
{[64]}\end{array}$ \\
\hline $\begin{array}{l}\text { Lung, Gastroin- } \\
\text { testinal } \\
\text { (I-IV) }\end{array}$ & $\begin{array}{c}69 \\
28 \mathrm{~F} / 21 \mathrm{M}\end{array}$ & $\begin{array}{c}\text { Age }=63.5 \pm \\
11.8 \\
\text { BMI }=\text { not } \\
\text { stated but } 87 \% \\
\text { moderate or } \\
\text { severe } \\
\text { malnutrition }\end{array}$ & Chemotherapy & $\begin{array}{c}600 \mathrm{kcal}: 2.2 \mathrm{~g} \\
\text { EPA } 33 \mathrm{~g} \\
\text { protein } \\
\mathrm{CNT}= \\
\text { isocaloric } \\
\text { supplement }\end{array}$ & 4 weeks & $\begin{array}{c}\text { N-3: } \downarrow \text { CRP (NS } \\
\text { due to } \\
\text { dropouts/death } \\
\text { only } 18 \text { in N-3 vs. } \\
25 \text { in control for } \\
\text { final analysis) }\end{array}$ & $\begin{array}{l}2014 \\
{[40]}\end{array}$ \\
\hline $\begin{array}{l}\text { NSCLC } \\
\text { (III) }\end{array}$ & $\begin{array}{c}40 \\
19 \mathrm{~F} / 21 \mathrm{M}\end{array}$ & $\begin{array}{c}\text { Age }=58.4 \pm \\
12.0 / 57.2 \pm 8.1 \\
\text { Body weight }= \\
77.1 \pm \\
14.6 / 64.7 \pm 7.4 \\
\text { BMI }=24.8 \pm \\
4.1 / 23.0 \pm 2.4\end{array}$ & $\begin{array}{l}\text { Cisplatin } \pm \\
\text { docetaxel or } \pm \\
\text { bevacizumab }+ \\
\text { concurrent } \\
\text { radiotherapy }\end{array}$ & $\begin{array}{c}600 \text { kcal: } 2.2 \mathrm{~g} \\
\text { EPA + } 1 \text { g DHA } \\
+32 \text { g protein } \\
\text { CNT }= \\
\text { isocaloric } \\
\text { supplement }\end{array}$ & 6 weeks & $\begin{array}{c}\text { N-3: weight } \\
\text { maintenance, NC } \\
\text { in CRP, IL-6, } \\
\text { TNFp55, albumin } \\
\text { and HLA-DR }\end{array}$ & $\begin{array}{l}2012 \\
{[53]}\end{array}$ \\
\hline $\begin{array}{l}\text { NSCLC } \\
\text { (III-IV) }\end{array}$ & $\begin{array}{l}84(44 / 40) \\
49 \mathrm{~F} / 43 \mathrm{M}\end{array}$ & $\begin{array}{c}\text { Age }=58.8 \pm \\
14 / 61.1 \pm 12.4 \\
\text { Body weight }= \\
60.4 \pm 11 / 64.7 \\
\pm 11 ; \text { BMI }= \\
24.2 \pm 3 / 25.2 \\
\pm 4 \\
\text { weight loss } \\
\text { before entry }= \\
8.8 \pm 8 \% / 7.4 \\
\pm 9 \%\end{array}$ & $\begin{array}{l}\text { Paclitaxel and } \\
\text { cisplatinum }\end{array}$ & $\begin{array}{c}300 \mathrm{kcal}: 1.1 \mathrm{~g} \\
\mathrm{EPA}+\text { protein } \\
\mathrm{CNT}= \\
\text { isocaloric } \\
\text { supplement }\end{array}$ & $\begin{array}{c}6 \text { weeks } \\
\text { supplement } \\
\text { and up to } 18 \\
\text { weeks } \\
\text { chemotherapy) }\end{array}$ & $\begin{array}{c}\text { N-3: weight } \\
\text { maintenance; } \downarrow \\
\text { CRP, TNF } \alpha \uparrow \uparrow \\
\text { protein intake } \\
\text { improved global } \\
\text { health status } \\
\text { (including fatigue } \\
\text { and improved } \\
\text { appetite); trend } \\
\text { towards } \\
\text { progression-free } \\
\text { survival Control: } \\
\text { weight loss, } \uparrow \\
\text { neuropathy }\end{array}$ & $\begin{array}{l}2014 \\
{[54]}\end{array}$ \\
\hline
\end{tabular}


Table 3. Cont.

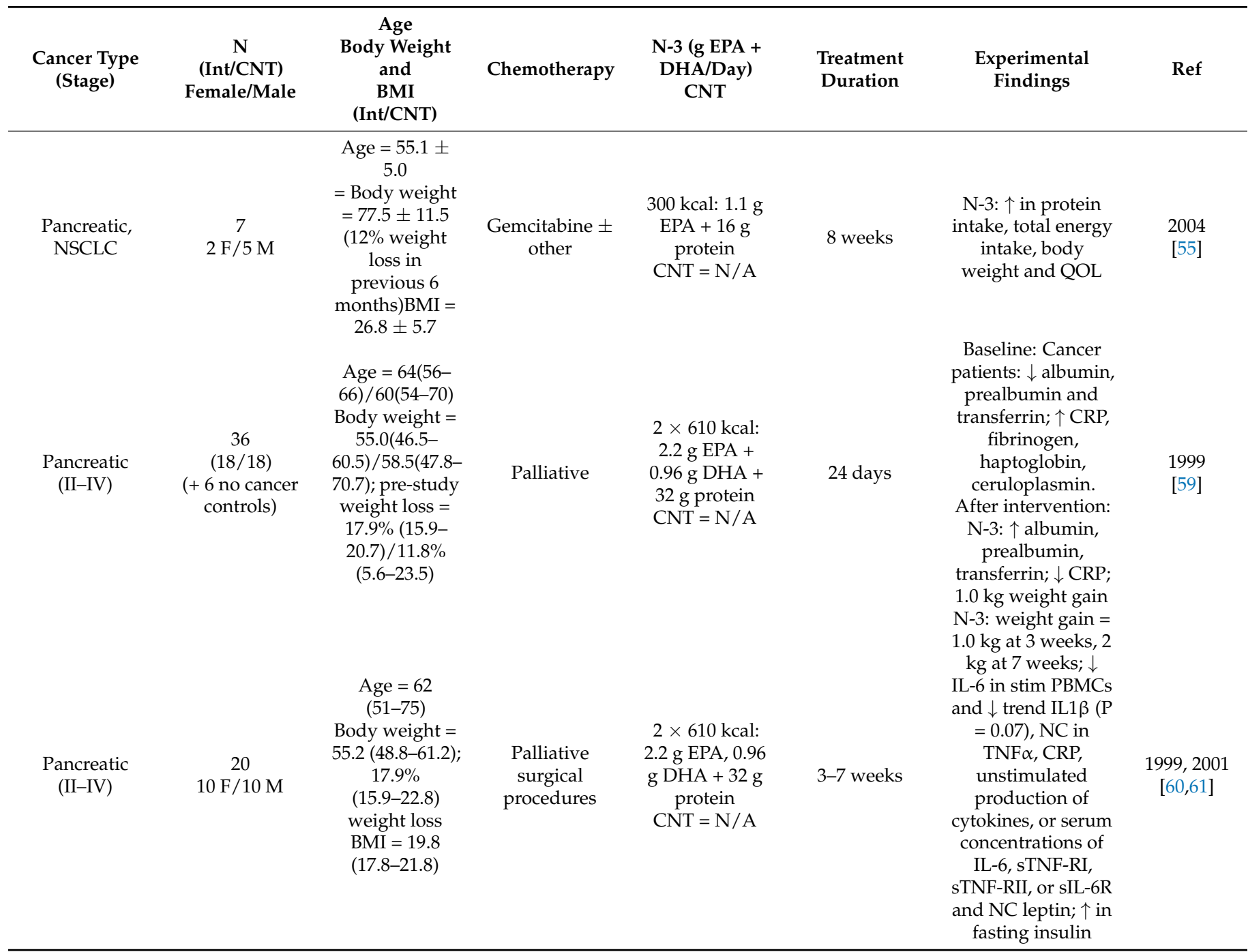

Abbreviations used: BMI, body mass index; CD, cluster of differentiation; CNT, control; CRP, C-reactive protein; DHA, docosahexaenoic acid; EPA, eicosapentaenoic acid; F, female; g, gram(s); GM-CSF, granulocyte macrophage colony-stimulating factor; HLADR, Human Leukocyte Antigen—DR; IFN $\gamma$, interferon gamma; IL, interleukin; Int, intervention group; kcal, kilocalorie; $\mathrm{kg}$, kilogram; $\mathrm{M}$, male; $\mathrm{N}$, number; N/A, not applicable; NC, no change, NS, non-significant; NSCLC, non-small-cell lung cancer; PBMC, peripheral blood mononuclear cell, PGE2, prostaglandin E2; PHA, phytohaemagglutinin; QOL, quality of life; RANTES, regulated on activation, normal T cell expressed and secreted (CCL5); Ref, reference; SF36, short form (36) health survey; TNF $\alpha$, tumor necrosis factor.

Table 4. Randomized controlled trials providing N-3 enteral or parenteral supplementation.

\begin{tabular}{|c|c|c|c|c|c|c|c|}
\hline $\begin{array}{c}\text { Cancer Type } \\
\text { (Stage) }\end{array}$ & $\begin{array}{c}\mathbf{N} \\
\text { (Int/CNT) } \\
\text { Female/Male }\end{array}$ & $\begin{array}{c}\text { Age } \\
\text { Body Weight } \\
\text { and } \\
\text { BMI } \\
\text { (Int/CNT) }\end{array}$ & Chemotherapy & $\begin{array}{l}\text { N-3 (EPA + } \\
\text { DHA/Day) } \\
\quad \text { CNT }\end{array}$ & $\begin{array}{l}\text { Treatment } \\
\text { Duration }\end{array}$ & $\begin{array}{l}\text { Experimental } \\
\text { Findings }\end{array}$ & Ref \\
\hline $\begin{array}{l}\text { Esophageal } \\
\text { (O-III) }\end{array}$ & $\begin{array}{c}27 \\
4 \mathrm{~F} / 23 \mathrm{M}\end{array}$ & $\begin{array}{c}\text { Age }=67 \pm \\
3 / 64 \pm 2\end{array}$ & $\mathrm{~N} / \mathrm{A}$ & $\begin{array}{c}150 \mathrm{mg} \\
\mathrm{n}-3 / 100 \mathrm{~mL} \\
\text { (up to max } 1.5 \\
\text { L/day }=2.25 \mathrm{~g} \text { ) } \\
+ \text { protein } \\
\text { CNT = EN }\end{array}$ & Day 0 and 8 & $\begin{array}{c}\text { N-3: NC IL-6 } \\
\text { between grps, } \downarrow \text { in } \\
\text { IL-8 (day } 1 \text { and 3) } \\
\text { and PGF1a (day 5) }\end{array}$ & $\begin{array}{l}2005 \\
{[65]}\end{array}$ \\
\hline
\end{tabular}


Table 4. Cont.

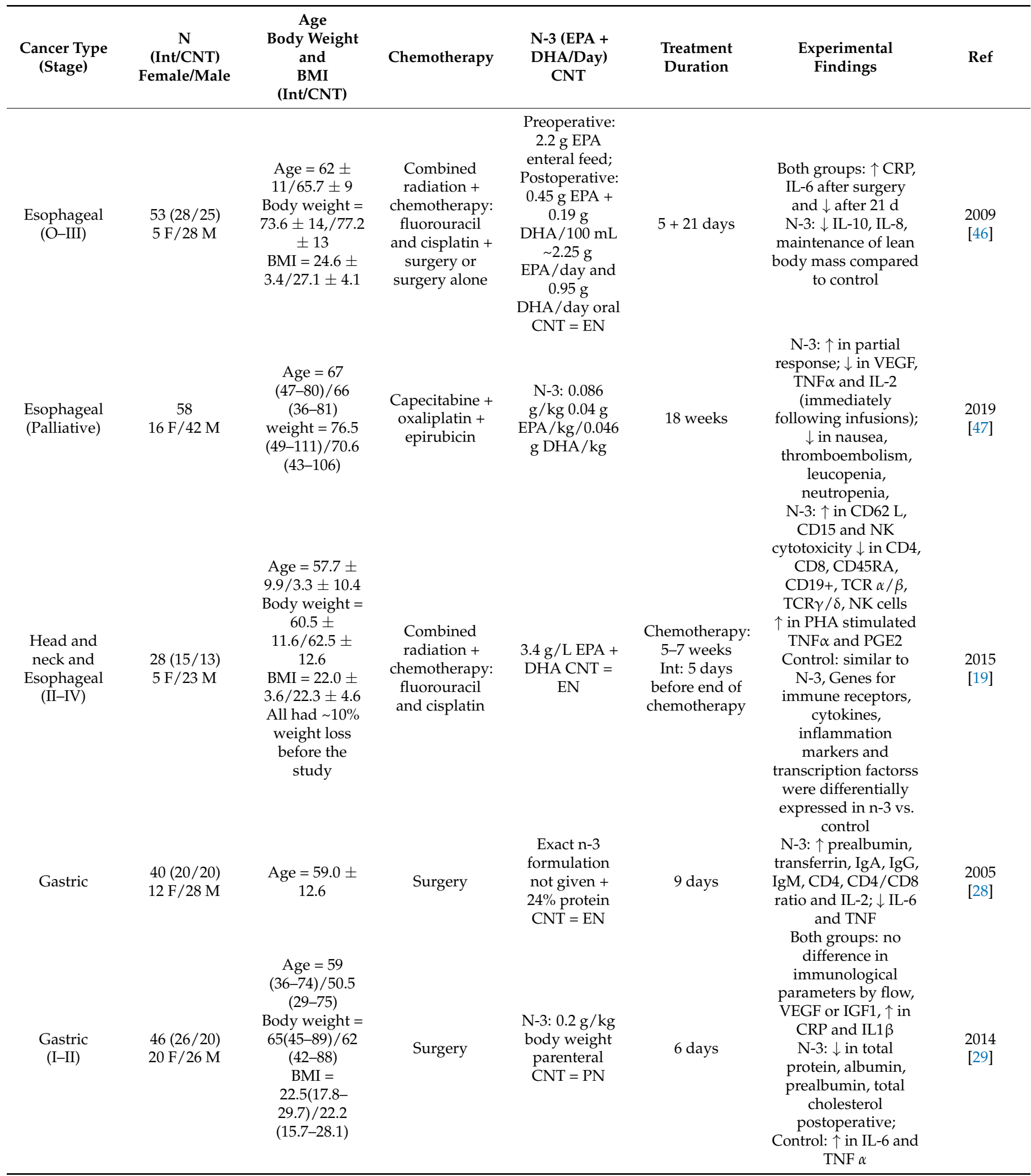


Table 4. Cont.

\begin{tabular}{|c|c|c|c|c|c|c|c|}
\hline $\begin{array}{c}\text { Cancer Type } \\
\text { (Stage) }\end{array}$ & $\begin{array}{c}\mathbf{N} \\
\text { (Int/CNT) } \\
\text { Female/Male }\end{array}$ & $\begin{array}{c}\text { Age } \\
\text { Body Weight } \\
\text { and } \\
\text { BMI } \\
\text { (Int/CNT) }\end{array}$ & Chemotherapy & $\begin{array}{l}\text { N-3 (EPA + } \\
\text { DHA/Day) } \\
\text { CNT }\end{array}$ & $\begin{array}{l}\text { Treatment } \\
\text { Duration }\end{array}$ & $\begin{array}{l}\text { Experimental } \\
\text { Findings }\end{array}$ & Ref \\
\hline Colorectal & $\begin{array}{l}\text { 200: } 4 \text { groups n } \\
=50 \text { control no } \\
\text { supplement, } \\
\text { control+ } \\
\text { supplement, } \\
\mathrm{N}-3 \text { before and } \\
\text { after surgery } \\
\text { and } \mathrm{N}-3 \\
\text { preoperative } \\
\text { only } \\
82 \mathrm{~F} / 118 \mathrm{M}\end{array}$ & $\begin{array}{c}\text { Age }=62.2 \pm \\
10.4 / 61.8 \pm \\
9.9 / 60.5 \pm \\
11.5 / 63.0 \pm 8.1 \\
\text { 20 Patients } \\
\text { with weight } \\
\text { loss }>10 \%\end{array}$ & Surgery & $\begin{array}{c}3.3 \mathrm{~g} \mathrm{~N}-3 / \mathrm{L} \\
\text { (patients } \\
\text { received } 25 \\
\mathrm{kcal} / \mathrm{kg} \text { body } \\
\text { weight) }+ \\
\text { protein } \\
\mathrm{CNT}=\mathrm{EN}\end{array}$ & $\begin{array}{c}7+7 \text { days } \\
\text { (pre }+ \text { post })\end{array}$ & $\begin{array}{l}\text { N-3: } \uparrow \text { phagocytic } \\
\text { ability of PMN } \\
\text { compared to controls } \\
\text { (did not drop } \\
\text { postoperative), } \uparrow \text { IL-6 } \\
\text { postoperative, but } \\
\text { lower compared to } \\
\text { control; } \downarrow \text { Delayed } \\
\text { hypersensitivity and } \\
\downarrow \text { infection in } \\
\text { supplemented } \\
\text { groups; NC in IGs }\end{array}$ & $\begin{array}{l}2002 \\
{[42]}\end{array}$ \\
\hline $\begin{array}{l}\text { Colorectal } \\
\text { and Rectal }\end{array}$ & $\begin{array}{c}42 \\
16 \mathrm{~F} / 25 \mathrm{M}\end{array}$ & $\begin{array}{c}\text { Age }=55.8 \pm \\
10.1 / 59.2 \pm \\
10.6 \\
\text { Body weight }= \\
63.5 \pm \\
8.9, / 65.4 \pm 9.2 \\
\text { BMI }=23.4 \pm \\
2.4 / 23.9 \pm 2.8\end{array}$ & Surgery & $\begin{array}{c}\mathrm{N}-3: 0.2 \mathrm{~g} / \mathrm{kg} \\
\text { body weight } \\
\text { parenteral } \\
\mathrm{CNT}=\mathrm{EN}\end{array}$ & 7 days & $\begin{array}{c}\text { Both groups } \uparrow \text { IL- } 6 \\
\text { on day of surgery } \\
\text { N-3: } \uparrow \text { CD } 4+\text { and } \downarrow \\
\text { IL-6 by day } 8 \text {; NS } \downarrow \\
\text { TNF }\end{array}$ & $\begin{array}{l}2008 \\
{[43]}\end{array}$ \\
\hline $\begin{array}{l}\text { Colorectal } \\
\text { and Rectal } \\
\text { (Duke B-C) }\end{array}$ & $\begin{array}{c}57 \\
24 \mathrm{~F} / 33 \mathrm{M}\end{array}$ & $\begin{array}{c}\text { Age }=69.8 \pm \\
10.5 / 70.8 \pm 6.4 \\
\mathrm{BMI}=22.9 \pm \\
3.1 / 23.2 \pm 3.6\end{array}$ & Surgery & $\begin{array}{c}\mathrm{N}-3: 0.2 \mathrm{~g} / \mathrm{kg} \\
\text { body weight } \\
\text { parenteral } \\
\mathrm{CNT}=\mathrm{EN}\end{array}$ & 7 days & $\begin{array}{l}\text { Both groups: } \downarrow \text { CD4 } \\
\text { on day } 8 \text { vs. day } 1 \\
\text { N-3: } \downarrow \text { CD8 day } 1 \text { and } \\
\text { day } 8 ; \downarrow \text { IL6 at day } 8 \\
\text { compared to control }\end{array}$ & $\begin{array}{l}2012 \\
{[44]}\end{array}$ \\
\hline Gastrointestinal & $\begin{array}{c}18 \\
7 \mathrm{~F} / 11 \mathrm{M}\end{array}$ & $\begin{array}{c}\text { Age }=69.8 \pm \\
2.7 / 65.4 \pm 4.2 \\
\text { Body weight }= \\
67.5 \pm 4.5 / 59.6 \\
\pm 3.025 \% \text { had } \\
\text { moderate to } \\
\text { severe protein } \\
\text { calorie } \\
\text { malnutrition }\end{array}$ & Surgery & $\begin{array}{c}\mathrm{N}-3: 3.98 \mathrm{~g}= \\
2.74 \mathrm{~g} \text { EPA, } \\
1.24 \mathrm{~g} \text { DHA } \\
\mathrm{CNT}=\mathrm{EN}\end{array}$ & 7 days & $\begin{array}{l}\text { N-3: } \downarrow \text { in ALT, AST } \\
\text { and Alkaline } \\
\text { phosphatase, } \downarrow \text { in } \\
\text { PGE2 production in } \\
\text { LPS stimulated cells }\end{array}$ & $\begin{array}{l}1997 \\
{[22]}\end{array}$ \\
\hline Gastrointestinal & $\begin{array}{c}50 \\
20 \mathrm{~F} / 30 \mathrm{M}\end{array}$ & $\begin{array}{c}\text { Age }=62.5 \pm \\
11.3 / 60.9 \pm \\
12.5 \\
11 \text { patients } \\
\text { with weight } \\
\text { loss }>10 \%\end{array}$ & Surgery & $\begin{array}{c}\mathrm{N}-3: 10.5 \% \text { of } \\
28 \% \text { fat in } 100 \\
\text { mL (patients } \\
\text { received } 25 \\
\text { kcal/kg body } \\
\text { weight) + } \\
\text { protein } \\
\text { CNT = EN }\end{array}$ & $\begin{array}{c}7+7 \text { days } \\
\text { (pre }+ \text { post })\end{array}$ & $\begin{array}{c}\text { N-3: } \uparrow \text { prealbumin } \\
\text { and retinol binding } \\
\text { protein and } \downarrow \text { IL-6, } \\
\text { IL-1RII and delayed } \\
\text { hypersensitivity at } \\
\text { day } 8, \text { NC in IGs. }\end{array}$ & $\begin{array}{l}1999 \\
{[23]}\end{array}$ \\
\hline Gastrointestinal & $\begin{array}{c}48 \\
17 \mathrm{~F} / 31 \mathrm{M}\end{array}$ & $\begin{array}{c}\text { Age }=55.2 \pm \\
12.1 / 52.6 \pm 9.8\end{array}$ & Surgery & $\begin{array}{c}146 \mathrm{kj} / \mathrm{kg} / \text { day: } \\
100 \mathrm{~mL}=125 \\
\mathrm{kcal}=0.08 \mathrm{~g} \\
\mathrm{EPA}+0.03 \mathrm{~g} \\
\mathrm{DHA}+4 \mathrm{~g} \\
\text { protein } \\
\mathrm{CNT}=\mathrm{EN}\end{array}$ & $\begin{array}{c}7+7 \text { days } \\
\text { (pre }+ \text { post })\end{array}$ & $\begin{array}{c}\text { Both groups: } \uparrow \text { PGE2 } \\
\text { and CRP } \\
\text { postoperatively } \\
\text { N-3: } \downarrow \text { PGE2, CRP } \\
\text { IL-6 and TNF by day } \\
8, \text { NS } \downarrow \text { in IL2, } \uparrow \\
\text { glutamine and } \\
\text { arginine; } \downarrow \text { in CD } 3+\text {, } \\
\text { CD4+ CD }+ \text { and NK } \\
\text { cells at day } 1 \text { and } \uparrow \\
\text { compared to baseline } \\
\text { and compared to } \\
\text { control at day } 8\end{array}$ & $\begin{array}{l}2001 \\
{[24]}\end{array}$ \\
\hline
\end{tabular}


Table 4. Cont.

\begin{tabular}{|c|c|c|c|c|c|c|c|}
\hline $\begin{array}{c}\text { Cancer Type } \\
\text { (Stage) }\end{array}$ & $\begin{array}{c}\mathrm{N} \\
(\text { Int/CNT) } \\
\text { Female/Male }\end{array}$ & $\begin{array}{c}\text { Age } \\
\text { Body Weight } \\
\text { and } \\
\text { BMI } \\
\text { (Int/CNT) }\end{array}$ & Chemotherapy & $\begin{array}{c}\text { N-3 (EPA + } \\
\text { DHA/Day) } \\
\text { CNT }\end{array}$ & $\begin{array}{l}\text { Treatment } \\
\text { Duration }\end{array}$ & $\begin{array}{l}\text { Experimental } \\
\text { Findings }\end{array}$ & Ref \\
\hline $\begin{array}{l}\text { Gastrointestinal } \\
\text { (II-III) }\end{array}$ & $\begin{array}{c}204 \\
73 \mathrm{~F} / 131 \mathrm{M}\end{array}$ & $\begin{array}{c}\text { Age }=56.3 \pm \\
10.1 / 58.2 \pm \\
11.0 \\
\text { Body weight }= \\
64.2 \pm \\
10.1 / 64.7 \pm \\
10.0 \mathrm{BMI}=22.8 \\
\pm 2.6 / 23.1 \pm \\
3.1\end{array}$ & Surgery & $\begin{array}{l}\mathrm{N}-3: 0.2 \mathrm{~g} / \mathrm{kg} \\
\text { body weight } \\
\text { parenteral } \\
\mathrm{CNT}=\mathrm{PN}\end{array}$ & 8 days & $\begin{array}{c}\text { N-3: } \downarrow \text { in CD8 and } \\
\text { NS } \downarrow \text { in IL- } 6 \text { and TNF } \\
\text { compared to control } \\
\text { at day } 8\end{array}$ & $\begin{array}{l}2010 \\
{[25]}\end{array}$ \\
\hline $\begin{array}{l}\text { Pancreatic } \\
\text { (Advanced) }\end{array}$ & $\begin{array}{c}50 \\
(20 \mathrm{~F} / 30 \mathrm{M})\end{array}$ & $\begin{array}{c}\text { Age }=68 \\
(40-83)\end{array}$ & Gemcitabine & $\begin{array}{c}\text { N-3: up to } 500 \\
\text { mL }(4.3-8.6 \mathrm{~g} \\
\text { of EPA + DHA) } \\
1 / \text { week } \\
\text { CNT = N/A }\end{array}$ & $\begin{array}{l}\text { Up to } 6 \text { cycles } \\
\text { (24 weeks) }\end{array}$ & $\begin{array}{l}\text { N-3: } \uparrow \text { in perceived } \\
\text { QOL; } 10 \% \uparrow \text { in global } \\
\text { health in } 47 \% \text { of } \\
\text { patients }\end{array}$ & 2015 [62] \\
\hline $\begin{array}{c}\text { Pancreatic/Bile } \\
\text { Duct }\end{array}$ & $\begin{array}{c}27 \\
(11 \mathrm{~F} / 16 \mathrm{M})\end{array}$ & $\begin{array}{c}\text { Age }=68.8 \pm \\
4.24\end{array}$ & $\begin{array}{c}\text { FOLFIRINOX; } \\
\text { gemcitabine + } \\
\text { nabpaclitaxel } \\
\text { or gemcitabine } \\
\text { + TS1; TSI } \\
\text { alone; } \\
\text { gemcitabine } \\
\text { alone or } \\
\text { cisplatin + } \\
\text { irinotecan }\end{array}$ & $\begin{array}{c}\text { N-3: } 2-4 \text { packs } \\
\text { (200 kcal/300 } \\
\text { mg N-3/pack) } \\
=0.60-1.20 \mathrm{~g} \\
\text { N3/day } \\
\text { CNT }=\text { N } / \mathrm{A}\end{array}$ & 8 weeks & $\begin{array}{c}\text { N-3: } \uparrow \text { in skeletal } \\
\text { muscle mass } \\
\text { compared to baseline; } \\
\uparrow \text { in NK cells at week } \\
\text { 8; trend towards } \\
\text { increase body weight }\end{array}$ & 2018 [63] \\
\hline
\end{tabular}

Abbreviations used: ALT, alanine aminotransferase; AST, aspartate transaminase; BMI, body mass index; CD, cluster of differentiation; CNT, control; CRP, C-reactive protein; DHA, docosahexaenoic acid; EPA, eicosapentaenoic acid; EN, standard enteral nutrition; F, female; g, gram(s); IG, immunoglobulin; IGF1, insulin-like growth factor 1; IL, interleukin; Int, intervention; kcal, kilocalorie; kg, kilogram; LPS, lipopolysaccharide; M, male; ml, millilitre; N, number; N/A, not applicable; NC, no change, NK, natural killer; NS, non-significant; PGE2, prostaglandin E2; PGF1a, prostaglandin F1a; PHA, phytohaemagglutinin; PMN, polymorphonuclear leukocytes; PN, standard parenteral nutrition; QOL, quality of life; Ref, reference; TCR, T-cell receptor; TNF $\alpha$, tumor necrosis factor; VEGF, vascular endothelial growth factor.

\subsection{N-3 Type, Amount Prescribed and Intervention Length}

Of the 57 independent studies assessed, two trials reported supplementation with DHA alone (in triglyceride form from an algal source [7] or titrated from fish oil [56], two studies employed EPA alone (as free fatty acids $[36,57]$ and 48 studies used a combination of EPA and DHA (derived from fish oil). An additional five studies reported only supplementing with EPA, yet based on the enteral supplement reported, these interventions likely included DHA but did not explicitly state it in the manuscript. Across the spectrum of studies, supplementation varied greatly in concentrations and intervention length. When the supplement was provided in an oral capsule form (Tables 1 and 2), supplementation ranged from 300 milligrams (mg) to 5 grams (g) of $\mathrm{n}-3$ fatty acids with $77 \%$ of the studies providing $2 \mathrm{~g}$ or less per day. Of the total supplementation, DHA concentrations ranged from 0 to $2 \mathrm{~g}$, with $86 \%$ of the studies providing $1 \mathrm{~g}$ or less per day. When capsules were provided concomitant with chemotherapy the intervention length was 6-24 weeks and equivalent to the duration of chemotherapy $[7-11,16,20,26,27,30-34,45,48-51,56,63]$. In the absence of chemotherapy, supplementation was provided for $1-6$ months [12-14,35,36,52,57]. Oral supplementation interventions were one week to 6 months in length, with over half of the studies ranging from 4 to 6 weeks in duration. Ten of the studies provided similar doses of EPA and DHA (2.2 and 0.9 g per day, respectively) with a range of 2-3.6 g of total n-3 per day (1.1-2.4 g EPA \pm 0.9-1.2 g DHA) and in one study, $4.9 \mathrm{~g}$ of EPA $+3.2 \mathrm{~g}$ of DHA per day (Table 3). The amount of $n-3$ fatty acids provided by enteral/parenteral nutrition was variably reported in the assessed studies; in some instances, $0.2 \mathrm{~g} \mathrm{n}-3$ per kilogram $(\mathrm{kg})$ of body weight and in others from 2.25 to $3.3 \mathrm{~g}$ per day (Table 4$)$. In these studies, the interventions were acute, generally limited to before and after surgery and were approximately $5-9$ days in duration. 


\subsection{Outcomes}

Reported outcomes varied across the studies but are grouped below into the following categories: (1) weight gain or maintenance, (2) serious adverse events including neuropathy and length of hospital stay, (3) immunological measures, (4) quality of life, (5) overall survival or progression-free survival and (6) additional parameters. Figure 2 provides an overall summary of the findings.

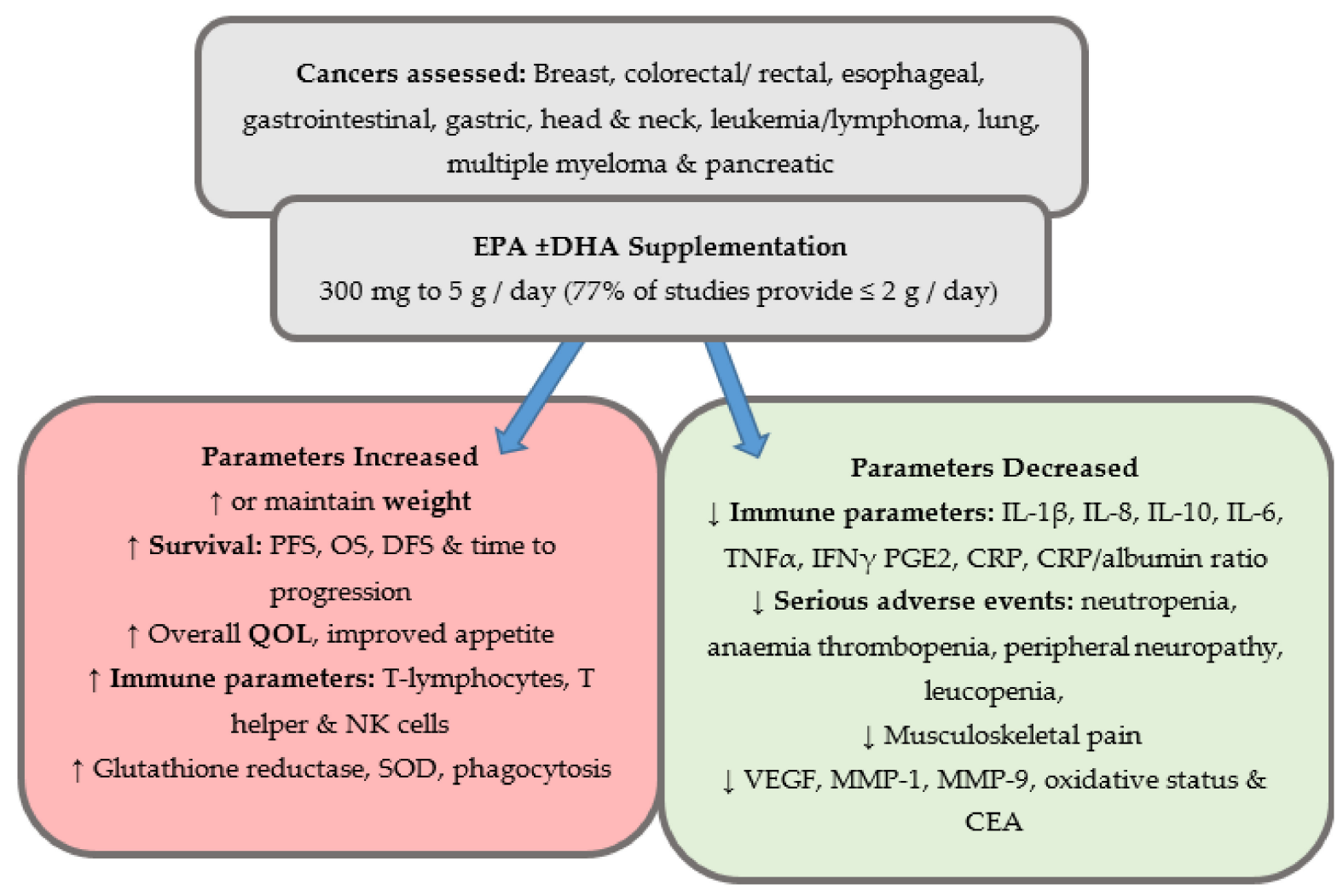

Figure 2. Overview of outcomes reported on the effects of $n-3$ supplementation compared to control or baseline status in clinical trials. Abbreviations used: CEA, carcinoembryonic antigen; CRP, C-reactive protein; DHA, docosahexaenoic acid, DFS, disease-free survival, EPA, eicosapentaenoic acid, IFN, interferon; IL, interleukin; MMP, matrix metalloproteinase, OS, overall survival, PGE2, prostaglandin E2; PFS, progression-free survival; QOL, quality of life; SOD, super oxide dismutase; TNF, tumor necrosis factor; VEGF, vascular endothelial growth factor.

\subsubsection{Weight}

Weight loss is a common side effect of both cancer and cancer therapies and has been reported on in many studies investigating the efficacy of $n-3$ supplementation particularly in advanced or palliative cancers and ones with defined pre-study weight loss. In general, oral liquid supplementation (which also included protein) was provided in instances where there was substantial weight loss prior to study entry and some evidence of malnutrition or cachexia. Compared to baseline status, $\mathrm{n}-3$ supplemented groups achieved weight gain in colorectal, gastric and pancreatic cancer studies $[31,37,55,59-61,63]$ or weight maintenance in one head and neck cancer study [18]. Two studies, one in advanced gastrointestinal cancer patients and one in stage III non-small-cell lung cancer patients observed improved weight maintenance in n-3 supplemented groups compared to control patients who were provided an isocaloric supplement that was not fortified with n-3 fatty acids $[39,53]$.

Capsule supplementation has been employed in three studies with palliative patients where weight maintenance was a reported study metric. In advanced lung cancer patients with systemic immune metabolic syndrome (SIMS, defined by presence of cachexia, anorexia, ECOG (Eastern Cooperative Oncology Group) status $>2$ and high CRP), the combination of $600 \mathrm{mg}$ EPA + DHA and celecoxib (non-steroidal anti-inflammatory drug) 
increased body weight by approximately $1.2 \mathrm{~kg}$ compared to the $\mathrm{n}-3$ capsules alone over a 6-week intervention [52]. Pancreatic cancer patients, who at study entry had lost approximately $13 \%$ body weight, stabilized their weight, and began to increase it by week 4 with EPA supplementation and the authors suggest that EPA could be a safe, effective anticachectic agent that could result in weight gain [57]. Finally, in a low-dose EPA + DHA (300 mg total per day) study of palliative pancreatic cancer patients, where two different sources were provided (fish oil or marine phospholipid capsules), similar effects were observed between the 2 groups suggesting the efficacy of n-3 fatty acids in weight stabilization [58].

Four studies reported weight maintenance or gain when n-3 fatty acids capsules were provided with neoadjuvant chemotherapy. In advanced lung cancer patients supplemented with $3.4 \mathrm{~g}$ of EPA + DHA concomitant with gemcitabine and cisplatin chemotherapy, body weight increased by $3.4 \mathrm{~kg}$ over the 66 day intervention [50]. Gastrointestinal patients supplemented with $700 \mathrm{mg}$ of EPA + DHA for 8 weeks with 5-fluorouracil and leucovorin chemotherapy gained an average of $1.7 \mathrm{~kg}$ over 8 weeks and although not significant to their baseline status, was significantly different from control patients who lost $2.5 \mathrm{~kg}$ average during this time frame [20]. Haidari et al. reported that colorectal cancer patients supplemented with $608 \mathrm{mg}$ of EPA + DHA resulted in weight maintenance over 8 weeks compared to control patients [27]. In colorectal cancer patients treated with xeloda, oxaliplatin, 5-fluorouracil and/or leucovorin therapy, it was observed that $600 \mathrm{mg}$ of EPA + DHA per day potentially prevented weight loss over 9 weeks when compared to standard of care control [32].

\subsubsection{Serious Adverse Events}

No studies reported serious adverse events attributable to n-3 supplementation, although in two instances with surgical patients for gastric and gastrointestinal cancers, there was improvement in overall postoperative recovery [29] and length of hospital stay [25], respectively, with parenteral n-3 nutrition. Lung cancer patients supplemented with up to $2.7 \mathrm{~g}$ of EPA + DHA were observed to have a better chemotherapy response rate during neoadjuvant therapy [49]. Common side effects of cytotoxic therapies used in breast cancer treatment include febrile neutropenia and neuropathy [66] and two studies observed a beneficial effect of supplementation on their side effects during neoadjuvant chemotherapy. Metastatic patients supplemented with $1.8 \mathrm{~g}$ of DHA per day had decreased neutropenia, anaemia and thrombopenia during cytotoxic chemotherapy [7]. Additionally, when women were supplemented with $1.2 \mathrm{~g}$ of EPA + DHA, Ghoreishi et al. observed a 70\% decreased risk of peripheral neuropathy incidence over 4 cycles of paclitaxel treatment [9]. These beneficial effects were also observed in palliative esophageal cancer patients, where EPA + DHA supplementation resulted in decreased nausea, thromboembolism, leucopenia, and neutropenia [47].

\subsubsection{Immunological Outcomes}

Enteral or parenteral nutrition is commonly employed for surgical interventions, resulting in elevated inflammation. All studies assessed in this review reported immunological modulations resulting from n-3 fatty acid-enriched enteral/parenteral nutrition. This included improved immune cell response [28,42,43,46]; decreased interleukin (IL)8 [46,65], IL-10 [46] and IL-6 (at time points 8-21 days post-surgery compared to control) $[23,24,28,29,42-44,46]$; modulation of functional capacity and gene expression of immune markers [19]; increased T-lymphocytes, T helper and natural killer (NK) cells [24]; modulated cytokine production [23,65]; decreased prostaglandin E2 (PGE2) [22] and reduced incidence of systemic inflammatory response syndrome [25,44]. C-reactive protein (CRP), a marker of inflammation that is often used as an indicator of poor prognosis, was routinely elevated post-surgery but found to decrease during n-3 supplementation in the days following $[24,46]$. 
Chemotherapy alters the immune response and inflammatory status [67] yet evidence of beneficial immunomodulation with $\mathrm{n}-3$ supplementation in non-surgical settings is limited. CRP was the most frequently assessed marker of inflammation, most often in advanced or palliative cancer patients, where it was observed that capsule/oral n-3 supplementation decreased CRP in head and neck [18], lung [40,52,54], gastrointestinal $[27,40,64]$, or pancreatic [64] cancers or maintained CRP levels during n-3 supplementation in breast [12,13], lung [50] or pancreatic cancer [57] compared to either baseline levels or increased CRP in non-supplemented controls (Supplementary Materials Table S1). Additionally, the CRP/albumin ratio, believed to be a predictor of overall survival in many cancers [68], was decreased during n-3 supplementation [32,33,48]. Other markers reported to be beneficially decreased during $n-3$ supplementation include IL-6 $[27,31,45,50]$, PGE2 [15,50], tumor necrosis factor alpha $(\mathrm{TNF} \alpha)[18,27,47,54]$ and interferon gamma (IFN $\gamma)[13,18,21]$. Purasiri et al. [35], assessed both localized and advanced colorectal cancer patients. In the patients with localized cancer, supplementation with $1 \mathrm{~g}$ EPA $+160 \mathrm{mg}$ DHA per day, short term until surgery, had no observed changes in immune parameters. However, in the advanced patients, where the amount of EPA + DHA increased to $2.1 \mathrm{~g}$ EPA and $320 \mathrm{mg}$ DHA daily for months 2 to 6 , a decrease in IL-1 $\beta$, IL-4, IL-6, TNF $\alpha$, and IFN $\gamma$ was observed. Interestingly, no changes in cytokine concentrations occurred in the first 2 months, and the authors suggested that long-term supplementation results in a significant decrease in circulating cytokines [35]. Additionally, neutrophil function during chemotherapy improved [20], cluster of differentiation (CD)4/CD8 ratio increased [14] and percent of CD4 + and CD8 + was maintained compared to elevation in controls [12].

\subsubsection{Quality of Life}

Changes in life quality have been frequently reported in clinical trial outcomes and often studies have employed the validated questionnaire from the European Organisation for Research and Treatment of Cancer-Quality of Life Questionnaire-C30 [69]. In capsule or oral $\mathrm{n}-3$ supplementation studies, improved perceived quality of life was reported in five studies $[26,37,45,54,55]$ and improved appetite reported twice-in colorectal and non-small-cell lung cancer studies [37,54]. Musculoskeletal pain is a well-documented side effect of aromatase inhibitors [70]. In women with previous chemotherapy or radiation and currently on aromatase inhibitors for breast cancer therapy, $1.4 \mathrm{~g}$ of $\mathrm{n}-3$ per day resulted in a $21.5 \%$ decrease on a pain scale after 30 days [13]. However, a second study in breast cancer patients on aromatase inhibitors found that supplementation with $3.3 \mathrm{~g}$ of EPA + DHA for 24 weeks only decreased pain significantly in obese patients [10,11].

\subsubsection{Survival}

Overall survival, progression-free survival and disease-free survival are key metrics reported in clinical trials. In the current review of the literature, studies investigating the benefits of $n-3$ supplementation via enteral/parenteral nutrition did not report on these metrics, likely due to the acute time frame of the interventions. However, a clinical benefit was observed in other studies. Stage III/IV lung cancer patients who received $1.1 \mathrm{~g}$ of EPA in a protein enriched oral supplement were reported to have a trend towards progression-free survival $(p<0.07)$ [54]. In metastatic patients with a variety of cancers, high doses of n-3 (5 g EPA + DHA combo) increased survival $(p<0.02)$, which was further increased if stratified between well-nourished and malnourished patients $(p<0.001)$ suggesting that malnutrition could be a predictor that affects $n-3$ supplementation prolonging survival [14]. Pancreatic patients generally have a median survival of 4.1 months, yet in a study by Wigmore et al., EPA supplementation (increasing doses up to a max $6 \mathrm{~g}$ per day for 12 weeks) increased the median survival to 6.8 months [57]. In a metastatic breast cancer study where all patients were DHA supplemented, stratification by amount of DHA incorporated into plasma showed that higher DHA incorporation was associated with longer time to progression ( 8.7 months vs. 3.5 months) and overall survival increased from 18 to 34 months [7]. One g of n-3 per day for 51 days 
increased overall survival (30.9 \pm 3.7 versus $25.9 \pm 3.6$ weeks, $p=0.05 ; \mathrm{HR}=0.41,95 \%$ CI: $0.20-0.84)$ and disease-free survival (28.5 \pm 3.3 versus $23.7 \pm 3.6, p=0.03 ; \mathrm{HR}=0.44$, 95\% CI: 0.22-0.87) in stage IIIB breast cancer patients [8]. Supplementation with $600 \mathrm{mg}$ $\mathrm{EPA}+\mathrm{DHA}$ increased time to progression in colorectal patients (20 months vs. 11 months for non-supplemented controls) [34] and overall long-term survival (at 465 days) compared to control in leukemia/lymphoma patients [48].

\subsubsection{Additional Parameters}

Two of the included studies assessed the Ki67 proliferation index. Ki67 in the tumor is a clinically relevant measure of efficacy in many clinical trials as it is expressed throughout the cell cycle $\left(G_{1}, S, G_{2}\right.$ and $M$ phases, but not in $\left.G_{0}\right)$ [71-73]. Darwito et al. found decreased Ki67 expression in breast cancer patients receiving $1 \mathrm{~g}$ per day of $\mathrm{n}-3$ supplement (39.2 \pm 5.3 versus $42.4 \pm 4.8, p=0.03$ ) [8], while there were no observed changes in the Ki67 proliferation index in patients with colorectal cancer and liver metastases when supplemented with $2 \mathrm{~g}$ of EPA per day [36]. Other experimentally relevant markers have been assessed in supplementation studies that suggest efficacy of n-3 fatty acids in modulating cancer outcomes including decreased vascular endothelial growth factor (VEGF) [47] and oxidative status [50], increased glutathione reductase, antioxidant status [16], superoxide dismutase $[16,20]$ and phagocytosis and $\mathrm{H}_{2} \mathrm{O}_{2}$ [20] in plasma or peripheral blood mononuclear cells and decreased VEGF [8], carcinoembryonic antigen [34] and gene expression of matrix metalloproteinase (MMP)-1 and MMP-9 [30] in tumors.

\section{Discussion}

The current scoping review brings together the evidence of over 50 clinical trials supporting the efficacy and antineoplastic effects of n-3 supplementation in a clinical setting. The goal of this review was to provide a broad overview of the evidence that is currently known on this topic and it is not intended to be a systematic review. Therefore, we have not provided a formal evaluation of the strength of the evidence or risk of bias for the studies included [74]. The evidence suggests that providing EPA and DHA (alone or in combination) can result in measurable clinical benefits across a spectrum of cancers. Although this research spans two decades, no clear guidelines for use as a therapeutic intervention have been defined. To establish recommendations, there is a need for future studies to focus on key factors that will strengthen the evidence currently available: (1) well-defined n-3 (EPA versus DHA) intervention, (2) clear dose based on preliminary trials, (3) expansion of outcomes assessed and (4) improved reporting of outcomes.

\subsection{N-3 Supplement Component and Dose Prescribed}

Benefits have been reported with both EPA and DHA, alone and in combination, at a range of doses from $300 \mathrm{mg}$ to $5 \mathrm{~g}$ per day. However, what the optimal combination of n-3 fatty acids is or what the dose should be is unclear. It is well established that EPA and DHA have both similar and unique mechanisms of action in cancer and other chronic diseases [75-79]. In an antineoplastic setting, while EPA more strongly inhibits arachidonic-derived prostaglandin production, DHA is known to modulate membrane lipid rafts, increase production of oxidative products and beneficially bind/activate nuclear receptors to a greater extent than EPA [76]. Currently, most studies used a combination of EPA and DHA, with EPA being the predominant fatty acid in the supplement, likely defined by the available supplements. Trial outcomes need to be clearly identified and then a single or mixed $n-3$ supplement used based on these parameters.

With respect to dose, it could be that regardless of the amount prescribed, there is a maximal level of incorporation within tumoral and tissue membranes. Achieving this through a personalized medicine approach and modifiable n-3 dose could be a consideration for future studies. As plasma is more easily accessible in a study setting compared to surgical tumor samples and plasma phospholipid membrane content of n-3 fatty acids correlates with tumor content [80], it is a metric that could be followed easily in clinic. 
Indeed, Bougnoux et al. found that patients with higher levels of plasma DHA had a longer time to progression and increased overall survival than patients with lower plasma levels, in a study where all patients received the same dose of $1.8 \mathrm{~g}$ DHA per day [7]. Additionally, while many of the studies included in this review reported that compliance was evaluated, confirmation through assessment of blood components or tissue fatty acid incorporation was reported in less than $20 \%$ of the studies $[7,9,12,19,20,35,36,49,50,57]$. Furthermore, consistent reporting of baseline would provide valuable information on incorporation and could be important in determination of clinical guidelines.

\subsection{Reported Outcomes}

Many outcomes have been reported in the literature, nonetheless there are three categories where future research and enhanced reporting would help strengthen the evidence currently available: (1) clinically relevant outcomes, (2) immune system modulation and (3) mechanistically relevant data.

\subsubsection{Clinically Relevant Outcomes}

Certain clinically relevant outcomes reporting the benefits of n-3 supplementation include weight loss and cancer cachexia. However, there exists a need for high-quality evidence for other metrics that are established clinical outcomes. Currently, only a few studies have reported on serious adverse events and the impact of EPA or DHA on chemotherapy associated toxicities. Taxanes are administered for many solid carcinomas including breast, ovarian, lung and pancreatic and neuropathies occur in up to $70 \%$ of these patients [81] although it is reported to be much lower with docetaxel compared to paclitaxel [66]. There are studies that have reported a beneficial decrease in taxane related neuropathies $[9,54,56]$. Interestingly, two of these studies had higher doses of DHA administered (compared to the EPA dose) and it would be of interest to determine whether DHA provides protection more efficaciously. Ki67 is used as a prognostic marker in several cancer types including breast cancer [82] and adrenocortical [83] yet was only reported in two studies in this review [8,36]. This is a quantifiable metric that would enable clinicians to establish concrete guidelines if it is routinely assessed in future studies. Additionally, many studies to date have focused on stage III, IV or palliative cancer patients and provide little data on progression-free survival, overall survival, or disease-free survival. How supplementation impacts cancer outcomes for earlier stage cancers is not known and this is a key metric that should be investigated in the future.

\subsubsection{Modulation of Immune Function}

The immune system, inflammation and cancer are inextricably linked. Progression of several cancers including pancreatic, gall bladder and esophageal results from chronic inflammation and prevalence of tumor-associated neutrophils that are associated with poor prognosis in many cancers including colorectal, melanoma and glioblastoma [84]. An estimated 40 to $60 \%$ of lung and gastrointestinal cancer patients exhibit elevated plasma concentrations of CRP [85] and this was consistently observed in the baseline data of the studies assessed herein. To counteract the inflammatory role in development or promotion of cancers, the beneficial role of n-3 fatty acids on the immune system of a cancer patient is becoming better defined. However, a comprehensive assessment of immune response has not been reported on in the current evidence. CRP data were reported in only $30 \%$ of the studies and only one study assessed time points other than baseline and end of study [41]. Future studies would benefit from a temporal assessment of changes in CRP over the course of chemotherapy as this molecule is often use as a surrogate marker of the inflammatory response. There is currently no $n-3$ supplementation study that has thoroughly detailed the effects of supplementation and the complex immune response of cytokines and immune cells that occur during the cancer trajectory. Profiling of both pro-inflammatory cytokines including IL-6, IL-1 $\beta$, IL-17, IL-23 and TNF $\alpha$ that are indicators of poor prognosis as well as anti-inflammatory cytokines including IL-4, IL-10, IL-13, and transforming growth factor 
(TGF) $\beta$ is recommended for future studies. Finally, dendritic cell activation and natural killer cell status are becoming increasingly recognized as important host responses through the cancer trajectory and have not been explored in the context of $n-3$ supplementation.

\subsubsection{Mechanistically Relevant Outcomes}

There exists a substantial base of strong preclinical evidence that details mechanisms and pathways involved in the anticancer actions of n-3 fatty acids. Mechanisms that have been identified include decreased cell proliferation, cell cycle progression and increased apoptosis and oxidative stress (reviewed in [4-6]). Pathways that are modulated through n-3 supplementation include the CD95 death receptor pathway, the Wnt/ $\beta$-catenin pathway, the mitogen-activated protein kinase (MAPK)/ERK pathway, the phosphoinositide 3-kinase (PI3K) pathway, the Janus kinase (JAK)-Signal transducer and activator of transcription (STAT) pathway and the nuclear factor (NF)- $\kappa B$ pathway [4]. Moving forward, it will be important to bridge the gap between preclinical and clinical evidence. Obtaining fresh tumor samples to properly assess mechanisms involved would greatly improve the strength of the evidence available. While this is not always practical in a clinical setting, future studies could also consider immunohistochemical assessment of fixed tumor samples or analysis of circulating tumor cells in blood.

\section{Conclusions}

The use of the n-3 fatty acids (EPA and/or DHA) as a therapeutic intervention in a clinical setting is backed by strong biological hypotheses and a large body of preclinical data. The current clinical evidence suggests that it could improve a variety of outcomes important to the patient and the disease process. There is, however, a need for larger, wellcontrolled, statistically powered studies with expanded reported outcomes. Furthermore, the amount and length of dose prescribed should be large enough and long enough to test the desired outcomes. Future studies should also include cancers where there are some promising preclinical data suggesting the efficacy of n-3 supplementation, such as ovarian, but for which there have been no clinical studies to date.

Supplementary Materials: The following are available online at https://www.mdpi.com/2072 $-6694 / 13 / 6 / 1206 / s 1$, Table S1: Characteristics of the C-reactive protein assessments included in clinical trials.

Author Contributions: All authors contributed to the original draft preparation, review and editing of this manuscript. Conceptualization, M.N. and C.J.F.; methodology, M.N. and C.J.F.; formal analysis, M.N., V.M., L.M.P. and C.J.F.; investigation, M.N., V.M., L.M.P. and C.J.F.; resources, M.N., V.M., L.M.P. and C.J.F.; data curation, M.N., V.M., L.M.P. and C.J.F.; writing-original draft preparation, M.N., V.M., L.M.P. and C.J.F.; writing-review and editing, M.N., V.M., L.M.P. and C.J.F.; visualization, M.N. and C.J.F.; supervision, C.J.F.; project administration, C.J.F.; funding acquisition, M.N. and C.J.F. All authors have read and agreed to the published version of the manuscript.

Funding: This research was funded by Canadian Institute of Health Sciences (CIHR) grant number RES0037745 and the Alberta Canola Producers Commission. Marnie Newell received a studentship from the Alberta Cancer Foundation.

Institutional Review Board Statement: Not applicable.

Informed Consent Statement: Not applicable.

Data Availability Statement: Not applicable.

Conflicts of Interest: The authors declare no conflict of interest.

\section{References}

1. Sung, H.; Ferlay, J.; Siegel, R.L.; Laversanne, M.; Soerjomataram, I.; Jemal, A.; Bray, F. Global cancer statistics 2020: GLOBOCAN estimates of incidence and mortality worldwide for 36 cancers in 185 countries. CA Cancer J. Clin. 2021. [CrossRef] [PubMed]

2. Turk, H.F.; Barhoumi, R.; Chapkin, R.S. Alteration of EGFR Spatiotemporal Dynamics Suppresses Signal Transduction. PLoS ONE 2012, 7, e39682. [CrossRef] 
3. Statistics Canada. Leading Causes of Death, Total Population. 2019. Available online: www150.statcan.gc.ca (accessed on 4 February 2021).

4. D'Eliseo, D.; Velotti, F. Omega-3 Fatty Acids and Cancer Cell Cytotoxicity: Implications for Multi-Targeted Cancer Therapy. J. Clin. Med. 2016, 5, 15. [CrossRef]

5. Liu, J.; Ma, D.W.L. The Role of n-3 Polyunsaturated Fatty Acids in the Prevention and Treatment of Breast Cancer. Nutrients 2014, 6, 5184-5223. [CrossRef] [PubMed]

6. Newell, M.; Baker, K.; Postovit, L.M.; Field, C.J. A Critical Review on the Effect of Docosahexaenoic Acid (DHA) on Cancer Cell Cycle Progression. Int. J. Mol. Sci. 2017, 18, 1784. [CrossRef] [PubMed]

7. Bougnoux, P.; Hajjaji, N.; Ferrasson, M.N.; Giraudeau, B.; Couet, C.; Le Floch, O. Improving outcome of chemotherapy of metastatic breast cancer by docosahexaenoic acid: A phase II trial. Br. J. Cancer 2009, 101, 1978-1985. [CrossRef]

8. Darwito, D.; Dharmana, E.; Riwanto, I.; Budijitno, S.; Suwardjo, S.; Purnomo, J.; Widodo, I.; Ghozali, A.; Aryandono, T.; Anwar, S.L. Effects of Omega-3 Supplementation on Ki-67 and VEGF Expression Levels and Clinical Outcomes of Locally Advanced Breast Cancer Patients Treated with Neoadjuvant CAF Chemotherapy: A Randomized Controlled Trial Report. Asian Pac. J. Cancer Prev. 2019, 20, 911-916. [CrossRef]

9. Ghoreishi, Z.; Esfahani, A.; Djazayeri, A.; Djalali, M.; Golestan, B.; Ayromlou, H.; Hashemzade, S.; Jafarabadi, M.A.; Montazeri, V.; Keshavarz, S.A.; et al. Omega-3 fatty acids are protective against paclitaxel-induced peripheral neuropathy: A randomized double-blind placebo controlled trial. BMC Cancer 2012, 12, 355. [CrossRef]

10. Hershman, D.L.; Unger, J.M.; Crew, K.D.; Awad, D.; Dakhil, S.R.; Gralow, J.; Greenlee, H.; Lew, D.L.; Minasian, L.M.; Till, C.; et al. Randomized Multicenter Placebo-Controlled Trial of Omega-3 Fatty Acids for the Control of Aromatase Inhibitor-Induced Musculoskeletal Pain: SWOG S0927. J. Clin. Oncol. 2015, 33, 1910-1917. [CrossRef] [PubMed]

11. Shen, S.; Unger, J.M.; Crew, K.D.; Till, C.; Greenlee, H.; Gralow, J.; Dakhil, S.R.; Minasian, L.M.; Wade, J.L., 3rd; Fisch, M.J.; et al. Omega-3 fatty acid use for obese breast cancer patients with aromatase inhibitor-related arthralgia (swog s0927). Breast Cancer Res. Treat. 2018, 172, 603-610. [CrossRef] [PubMed]

12. Da Silva Paixão, E.M.; de M. Oliveira, A.C.; Pizato, N.; Muniz-Junqueira, M.I.; Magalhães, K.G.; Yoshio Nakano, E.; Ito, M.K. The effects of epa and DHA enriched fish oil on nutritional and immunological markers of treatment naïve breast cancer patients: A randomized double-blind controlled trial. Nutr. J. 2017, 16, 71.

13. Martínez, N.; Herrera, M.; Frías, L.; Provencio, M.; Pérez-Carrión, R.; Díaz, V.; Morse, M.; Crespo, M.C. A combination of hydroxytyrosol, omega-3 fatty acids and curcumin improves pain and inflammation among early stage breast cancer patients receiving adjuvant hormonal therapy: Results of a pilot study. Clin. Transl. Oncol. 2019, 21, 489-498. [CrossRef] [PubMed]

14. Gogos, C.A.; Ginopoulos, P.; Salsa, B.; Apostolidou, E.; Zoumbos, N.C.; Kalfarentzos, F. Dietary omega-3 polyunsaturated fatty acids plus vitamin e restore immunodeficiency and prolong survival for severely ill patients with generalized malignancy: A randomized control trial. Cancer 1998, 82, 395-402. [CrossRef]

15. Faber, J.; Berkhout, M.; Fiedler, U.; Avlar, M.; Witteman, B.; Vos, A.; Henke, M.; Garssen, J.; Van Helvoort, A.; Otten, M.; et al. Rapid EPA and DHA incorporation and reduced PGE2 levels after one week intervention with a medical food in cancer patients receiving radiotherapy, a randomized trial. Clin. Nutr. 2013, 32, 338-345. [CrossRef]

16. Mansara, P.; Ketkar, M.; Deshpande, R.; Chaudhary, A.; Shinde, K.; Kaul-Ghanekar, R. Improved antioxidant status by omega-3 fatty acid supplementation in breast cancer patients undergoing chemotherapy: A case series. J. Med. Case Rep. 2015, 9, 148. [CrossRef] [PubMed]

17. Hanai, N.; Terada, H.; Hirakawa, H.; Suzuki, H.; Nishikawa, D.; Beppu, S.; Hasegawa, Y. Prospective randomized investigation implementing immunonutritional therapy using a nutritional supplement with a high blend ratio of $\omega$-3 fatty acids during the perioperative period for head and neck carcinomas. Jpn. J. Clin. Oncol. 2018, 48, 356-361. [CrossRef] [PubMed]

18. Solís-Martínez, O.; Plasa-Carvalho, V.; Phillips-Sixtos, G.; Trujillo-Cabrera, Y.; Hernández-Cuellar, A.; Queipo-García, G.E.; Meaney-Mendiolea, E.; Ceballos-Reyes, G.M.; Fuchs-Tarlovsky, V. Effect of Eicosapentaenoic Acid on Body Composition and Inflammation Markers in Patients with Head and Neck Squamous Cell Cancer from a Public Hospital in Mexico. Nutr. Cancer 2018, 70, 663-670. [CrossRef] [PubMed]

19. Talvas, J.; Garrait, G.; Goncalves-Mendez, N.; Rouanet, J.; Vergnaud-Gauduchon, J.; Kwiatkowski, F.; Bachmann, P.; Bouteloup, C.; Bienvenu, J.; Vasson, M.-P. Immunonutrition stimulates immune functions and antioxidant defense capacities of leukocytes in radiochemotherapy-treated head \& neck and esophageal cancer patients: A double-blind randomized clinical trial. Clin. Nutr. 2015, 34, 810-817. [CrossRef]

20. Bonatto, S.J.R.; Oliveira, H.H.P.; Nunes, E.A.; Pequito, D.; Iagher, F.; Coelho, I.; Naliwaiko, K.; Kryczyk, M.; Brito, G.A.P.; Repka, J.; et al. Fish Oil Supplementation Improves Neutrophil Function during Cancer Chemotherapy. Lipids 2012, 47, 383-389. [CrossRef]

21. Gómez-Candela, C.; Sanz, M.V.; Horrisberger, A.; Kohen, V.L.; Bermejo, L.M.; Auñón, P.Z. Efficacy evaluation of an oral powder supplement enriched with eicosapentaenoic acid in cancer patients. Nutr. Hosp. 2011, 26, 1385-1393.

22. Swails, W.S.; Kenler, A.S.; Driscoll, D.F.; DeMichele, S.J.; Babineau, T.J.; Utsunamiya, T.; Chavali, S.; Forse, R.A.; Bistrian, B.R. Effect of a Fish Oil Structured Lipid-Based Diet on Prostaglandin Release from Mononuclear Cells in Cancer Patients after Surgery. J. Parenter. Enter. Nutr. 1997, 21, 266-274. [CrossRef] [PubMed]

23. Gianotti, L.; Braga, M.; Fortis, C.; Soldini, L.; Vignali, A.; Colombo, S.; Radaelli, G.; Di Carlo, V. A Prospective, Randomized Clinical Trial on Perioperative Feeding with an Arginine-, Omega-3 Fatty Acid-, and RNA-Enriched Enteral Diet: Effect on Host Response and Nutritional Status. J. Parenter. Enter. Nutr. 1999, 23, 314-320. [CrossRef] 
24. Wu, G.H.; Zhang, Y.-W.; Wu, Z.-H. Modulation of postoperative immune and inflammatory response by immune-enhancing enteral diet in gastrointestinal cancer patients. World J. Gastroenterol. 2001, 7, 357-362. [CrossRef]

25. Jiang, Z.M.; Wilmore, D.W.; Wang, X.R.; Wei, J.M.; Zhang, Z.T.; Gu, Z.Y.; Wang, S.; Han, S.M.; Jiang, H.; Yu, K. Randomized clinical trial of intravenous soybean oil alone versus soybean oil plus fish oil emulsion after gastrointestinal cancer surgery. BJS 2010, 97, 804-809. [CrossRef] [PubMed]

26. Camargo, C.D.Q.; Mocellin, M.C.; Brunetta, H.S.; Chagas, T.R.; Fabre, M.E.S.; Trindade, E.; Da Silva, E.L.; Nunes, E.A. Fish oil decreases the severity of treatment-related adverse events in gastrointestinal cancer patients undergoing chemotherapy: A randomized, placebo-controlled, triple-blind clinical trial. Clin. Nutr. ESPEN 2019, 31, 61-70. [CrossRef] [PubMed]

27. Haidari, F.; Abiri, B.; Iravani, M.; Ahmadi-Angali, K.; Vafa, M. Randomized Study of the Effect of Vitamin D and Omega-3 Fatty Acids Cosupplementation as Adjuvant Chemotherapy on Inflammation and Nutritional Status in Colorectal Cancer Patients. J. Diet. Suppl. 2020, 17, 384-400. [CrossRef] [PubMed]

28. Chen, D.W.; Fei, Z.W.; Zhang, Y.C.; Ou, J.M.; Xu, J. Role of Enteral Immunonutrition in Patients with Gastric Carcinoma Undergoing Major Surgery. Asian J. Surg. 2005, 28, 121-124. [CrossRef]

29. Wei, Z.; Wang, W.; Chen, J.; Yang, D.; Yan, R.; Cai, Q. A prospective, randomized, controlled study of [omega]-3 fish oil fat emulsion-based parenteral nutrition for patients following surgical resection of gastric tumors. Nutr. J. 2014, 13, 25. [CrossRef]

30. Khojastehfard, M.; Dolatkhah, H.; Somi, M.-H.; Ahmad, S.N.S.; Estakhri, R.; Sharifi, R.; NaghiZadeh, M.; Rahmati-Yamchi, M. The Effect of Oral Administration of PUFAs on the Matrix Metalloproteinase Expression in Gastric Adenocarcinoma Patients Undergoing Chemotherapy. Nutr. Cancer 2019, 71, 444-451. [CrossRef]

31. Feijó, P.M.; Rodrigues, V.D.; Viana, M.S.; Dos Santos, M.P.; Abdelhay, E.; Viola, J.P.; De Pinho, N.B.; Martucci, R.B. Effects of $\omega-3$ supplementation on the nutritional status, immune, and inflammatory profiles of gastric cancer patients: A randomized controlled trial. Nutrition 2019, 61, 125-131. [CrossRef]

32. Mocellin, M.C.; Silva, J.D.A.P.E.; Camargo, C.D.Q.; Fabre, M.E.; Gevaerd, S.; Naliwaiko, K.; Moreno, Y.M.F.; Nunes, E.A.; Trindade, E.B. Fish Oil Decreases C-Reactive Protein/Albumin Ratio Improving Nutritional Prognosis and Plasma Fatty Acid Profile in Colorectal Cancer Patients. Lipids 2013, 48, 879-888. [CrossRef]

33. Pastore-Silva, J.; Trindade, E.; Fabre, M.; Menegotto, V.M.; Gevaerd, S.; Buss, Z.; Frode, T.S. Fish oil supplement alters markers of inflammatory and nutritional status in colorectal cancer patients. Nutr. Cancer 2012, 64, 267-273. [CrossRef] [PubMed]

34. Camargo, C.D.Q.; Mocellin, M.C.; Silva, J.D.A.P.; Fabre, M.E.; Nunes, E.A.; Trindade, E.B. Fish oil supplementation during chemotherapy increases posterior time to tumor progression in colorectal cancer. Nutr. Cancer 2016, 68, 70-76. [CrossRef] [PubMed]

35. Purasiri, P.; Murray, A.; Richardson, S.; Heys, S.D.; Horrobin, D.; Eremin, O. Modulation of Cytokine Production In Vivo by Dietary Essential Fatty Acids in Patients with Colorectal Cancer. Clin. Sci. 1994, 87, 711-717. [CrossRef] [PubMed]

36. Cockbain, A.J.; Volpato, M.; Race, A.D.; Munarini, A.; Fazio, C.; Belluzzi, A.; Loadman, P.M.; Toogood, G.J.; Hull, M.A. Anticolorectal cancer activity of the omega-3 polyunsaturated fatty acid eicosapentaenoic acid. Gut 2014, 63, 1760-1768. [CrossRef]

37. Trabal, J.; Leyes, P.; Forga, M.; Maurel, J. Potential usefulness of an EPA-enriched nutritional supplement on chemotherapy tolerability in cancer patients without overt malnutrition. Nutr. Hosp. 2010, 25, 736-ss740.

38. Kemen, M.; Senkal, M.; Homann, H.-H.; Mumme, A.; Dauphin, A.-K.; Baier, J.; Windeler, J.; Neumann, H.; Zumtobel, V. Early postoperative enteral nutrition with arginine-omega-3 fatty acids and ribonucleic acid-supplemented diet versus placebo in cancer patients: An immunologic evaluation of impact. Crit. Care Med. 1995, 23, 652-659. [CrossRef] [PubMed]

39. Persson, C.; Glimelius, B.; Rönnelid, J.; Nygren, P. Impact of fish oil and melatonin on cachexia in patients with advanced gastrointestinal cancer: A randomized pilot study. Nutrition 2005, 21, 170-178. [CrossRef] [PubMed]

40. Pastore, C.A.; Orlandi, S.P.; Gonzalez, M.C. Introduction of an Omega-3 Enriched Oral Supplementation for Cancer Patients Close to the First Chemotherapy: May It Be a Factor for Poor Compliance? Nutr. Cancer 2014, 66, 1285-1292. [CrossRef] [PubMed]

41. Read, J.A.; Beale, P.J.; Volker, D.H.; Smith, N.; Childs, A.; Clarke, S.J. Nutrition intervention using an eicosapentaenoic acid (EPA)-containing supplement in patients with advanced colorectal cancer. Effects on nutritional and inflammatory status: A phase II trial. Support. Care Cancer 2007, 15, 301-307. [CrossRef]

42. Braga, M.; Gianotti, L.; Vignali, A.; Di Carlo, V. Preoperative oral arginine and n-3 fatty acid supplementation improves the immunometabolic host response and outcome after colorectal resection for cancer. Surgery 2002, 132, 805-814. [CrossRef] [PubMed]

43. Liang, B.; Wang, S.; Ye, Y.-J.; Yang, X.-D.; Wang, Y.-L.; Qu, J.; Xie, Q.-W.; Yin, M.-J. Impact of postoperative omega-3 fatty acid-supplemented parenteral nutrition on clinical outcomes and immunomodulations in colorectal cancer patients. World $J$. Gastroenterol. 2008, 14, 2434-2439. [CrossRef]

44. Zhu, M.-W.; Tang, D.-N.; Hou, J.; Wei, J.-M.; Hua, B.; Sun, J.-H.; Cui, H.-Y. Impact of fish oil enriched total parenteral nutrition on elderly patients after colorectal cancer surgery. Chin. Med J. 2012, 125, 178-181. [PubMed]

45. Golkhalkhali, B.; Rajandram, R.; Paliany, A.S.; Ho, G.F.; Ishak, W.Z.W.; Johari, C.S.; Chin, K.F. Strain-specific probiotic (microbial cell preparation) and omega-3 fatty acid in modulating quality of life and inflammatory markers in colorectal cancer patients: A randomized controlled trial. Asia-Pac. J. Clin. Oncol. 2018, 14, 179-191. [CrossRef]

46. Ryan, A.M.; Reynolds, J.V.; Healy, L.; Byrne, M.; Moore, J.; Brannelly, N.; McHugh, A.; McCormack, D.; Flood, P. Enteral Nutrition Enriched with Eicosapentaenoic Acid (EPA) Preserves Lean Body Mass Following Esophageal Cancer Surgery: Results of a Double-Blinded Randomized Controlled Trial. Ann. Surg. 2009, 249, 355-363. [CrossRef] [PubMed] 
47. Eltweri, A.M.; Thomas, A.L.; Chung, W.Y.; Morgan, B.; Thompson, J.; Dennison, A.R.; Bowrey, D.J. The Effect of Supplementary Omegaven ${ }^{\circledR}$ on the Clinical Outcome of Patients with Advanced Esophagogastric Adenocarcinoma Receiving Palliative Epirubicin, Oxaliplatin, and Capecitabine Chemotherapy: A Phase II clinical trial. Anticancer. Res. 2019, 39, 853-861. [CrossRef] [PubMed]

48. Chagas, T.R.; Borges, D.S.; De Oliveira, P.F.; Mocellin, M.C.; Barbosa, A.M.; Camargo, C.Q.; Del Moral, J.Â.G.; Poli, A.; Calder, P.C.; Trindade, E.B.S.M.; et al. Oral fish oil positively influences nutritional-inflammatory risk in patients with haematological malignancies during chemotherapy with an impact on long-term survival: A randomised clinical trial. J. Hum. Nutr. Diet. 2017, 30, 681-692. [CrossRef]

49. Murphy, R.A.; Mourtzakis, M.; Chu, Q.S.C.; Baracos, V.E.; Reiman, T.; Mazurak, V.C. Supplementation with fish oil increases first-line chemotherapy efficacy in patients with advanced nonsmall cell lung cancer. Cancer 2011, 117, 3774-3780. [CrossRef]

50. Finocchiaro, C.; Segre, O.; Fadda, M.; Monge, T.; Scigliano, M.; Schena, M.; Tinivella, M.; Tiozzo, E.; Catalano, M.G.; Pugliese, M.; et al. Effect of n-3 fatty acids on patients with advanced lung cancer: A double-blind, placebo-controlled study. Br. J. Nutr. 2012, 108, 327-333. [CrossRef]

51. Yan, L.; Ren-gang, C.; San-zou, W.; Han-guo, H.; Fei, S.; Chun-hui, Y.; Lu, Y.; Chen, R.-G.; Wei, S.-Z.; Hu, H.-G.; et al. Effect of omega 3 fatty acids on c-reactive protein and interleukin-6 in patients with advanced nonsmall cell lung cancer. Medicine 2018, $97,1-4$.

52. Cerchietti, L.C.; Navigante, A.H.; Castro, M.A. Effects of Eicosapentaenoic and Docosahexaenoic n-3 Fatty Acids from Fish Oil and Preferential Cox-2 Inhibition on Systemic Syndromes in Patients with Advanced Lung Cancer. Nutr. Cancer 2007, 59, 14-20. [CrossRef]

53. Van Der Meij, B.S.; Langius, J.A.E.; Spreeuwenberg, M.D.; Slootmaker, S.M.; A Paul, M.; Smit, E.F.; Van Leeuwen, P.A.M. Oral nutritional supplements containing n-3 polyunsaturated fatty acids affect quality of life and functional status in lung cancer patients during multimodality treatment: An RCT. Eur. J. Clin. Nutr. 2012, 66, 399-404. [CrossRef] [PubMed]

54. Sánchez-Lara, K.; Turcott, J.G.; Juárez-Hernández, E.; Nuñez-Valencia, C.; Villanueva, G.; Guevara, P.; De la Torre-Vallejo, M.; Mohar, A.; Arrieta, O. Effects of an oral nutritional supplement containing eicosapentaenoic acid on nutritional and clinical outcomes in patients with advanced non-small cell lung cancer: Randomised trial. Clin. Nutr. 2014, 33, 1017-1023. [CrossRef] [PubMed]

55. Bauer, J.D.; Capra, S. Nutrition intervention improves outcomes in patients with cancer cachexia receiving chemotherapy? A pilot study. Support. Care Cancer 2005, 13, 270-274. [CrossRef] [PubMed]

56. Maschio, M.; Zarabla, A.; Maialetti, A.; Marchesi, F.; Giannarelli, D.; Gumenyuk, S.; Pisani, F.; Renzi, D.; Galiè, E.; Mengarelli, A. Prevention of Bortezomib-Related Peripheral Neuropathy with Docosahexaenoic Acid and $\alpha$-Lipoic Acid in Patients with Multiple Myeloma: Preliminary Data. Integr. Cancer Ther. 2018, 17, 1115-1124. [CrossRef]

57. Wigmore, S.J.; Barber, M.D.; Ross, J.A.; Tisdale, M.J.; Fearon, K.C.H. Effect of Oral Eicosapentaenoic Acid on Weight Loss in Patients with Pancreatic Cancer. Nutr. Cancer 2000, 36, 177-184. [CrossRef] [PubMed]

58. Werner, K.; De Gaudry, D.K.; Taylor, L.A.; Keck, T.; Unger, C.; Hopt, U.T.; Massing, U. Dietary supplementation with n-3fatty acids in patients with pancreatic cancer and cachexia: Marine phospholipids versus fish oil—A randomized controlled double-blind trial. Lipids Heal. Dis. 2017, 16, 104. [CrossRef]

59. Barber, M.D.; Ross, J.A.; Preston, T.; Shenkin, A.; Fearon, K.C.H. Fish oil-enriched nutritional supplement attenuates progression of the acute-phase response in weight-losing patients with advanced pancreatic cancer. J. Nutr. 1999, 129, 1120-1125. [CrossRef]

60. Barber, M.D.; Ross, J.A.; Voss, A.C.; Tisdale, M.J.; Fearon, K.C.H. The effect of an oral nutritional supplement enriched with fish oil on weight-loss in patients with pancreatic cancer. Br. J. Cancer 1999, 81, 80-86. [CrossRef]

61. Barber, M.D.; Fearon, K.C.H.; Tisdale, M.J.; McMillan, D.C.; Ross, J.A. Effect of a Fish Oil-Enriched Nutritional Supplement on Metabolic Mediators in Patients with Pancreatic Cancer Cachexia. Nutr. Cancer 2001, 40, 118-124. [CrossRef]

62. Arshad, A.; Chung, W.; Isherwood, J.; Mann, C.; Al-Leswas, D.; Steward, W.; Metcalfe, M.; Dennison, A. Cellular and plasma uptake of parenteral omega-3 rich lipid emulsion fatty acids in patients with advanced pancreatic cancer. Clin. Nutr. 2014, 33, 895-899. [CrossRef] [PubMed]

63. Abe, K.; Uwagawa, T.; Haruki, K.; Takano, Y.; Onda, S.; Sakamoto, T.; Gocho, T.; Yanaga, K. Effects of $\omega$-3 Fatty Acid Supplementation in Patients with Bile Duct or Pancreatic Cancer Undergoing Chemotherapy. Anticancer. Res. 2018, 38, $2369-2375$. [CrossRef] [PubMed]

64. Shirai, Y.; Okugawa, Y.; Hishida, A.; Ogawa, A.; Okamoto, K.; Shintani, M.; Morimoto, Y.; Nishikawa, R.; Yokoe, T.; Tanaka, K.; et al. Fish oil-enriched nutrition combined with systemic chemotherapy for gastrointestinal cancer patients with cancer cachexia. Sci. Rep. 2017, 7, 4826. [CrossRef]

65. Aiko, S.; Yoshizumi, Y.; Tsuwano, S.; Shimanouchi, M.; Sugiura, Y.; Maehara, T. The Effects of Immediate Enteral Feeding with a Formula Containing High Levels of $\omega-3$ Fatty Acids in Patients after Surgery for Esophageal Cancer. J. Parenter. Enter. Nutr. 2005, 29, 141-147. [CrossRef] [PubMed]

66. Ho, M.Y.; Mackey, J.R. Presentation and management of docetaxel-related adverse effects in patients with breast cancer. Cancer Manag. Res. 2014, 6, 253-259. [CrossRef]

67. Van Der Most, R.G.; Currie, A.J.; Robinson, B.W.S.; A Lake, R. Decoding dangerous death: How cytotoxic chemotherapy invokes inflammation, immunity or nothing at all. Cell Death Differ. 2008, 15, 13-20. [CrossRef] [PubMed] 
68. Zhou, T.; Zhan, J.; Hong, S.; Hu, Z.; Fang, W.; Qin, T.; Ma, Y.; Yang, Y.; He, X.; Zhao, Y.; et al. Ratio of C-Reactive Protein/Albumin Is An Inflammatory Prognostic Score for Predicting Overall Survival of Patients with Small-Cell Lung Cancer. Sci. Rep. 2015, 5, 10481. [CrossRef] [PubMed]

69. Aaronson, N.K.; De Haes, J.C.J.M.; Kaasa, S.; Klee, M.; Osoba, D.; Razavi, D.; Rofe, P.B.; Schraub, S.; Sneeuw, K.; Sullivan, M.; et al. The European Organization for Research and Treatment of Cancer QLQ-C30: A Quality-of-Life Instrument for Use in International Clinical Trials in Oncology. J. Natl. Cancer Inst. 1993, 85, 365-376. [CrossRef]

70. Henry, N.L.; Jacobson, J.A.; Banerjee, M.; Hayden, J.; Smerage, J.B.; Van Poznak, C.; Storniolo, A.M.; Stearns, V.; Hayes, D.F. A prospective study of aromatase inhibitor-associated musculoskeletal symptoms and abnormalities on serial high-resolution wrist ultrasonography. Cancer 2010, 116, 4360-4367. [CrossRef] [PubMed]

71. Dowsett, M.; Nielsen, T.O.; A’Hern, R.; Bartlett, J.; Coombes, R.C.; Cuzick, J.; Ellis, M.; Henry, N.L.; Hugh, J.C.; Lively, T.; et al. Assessment of Ki67 in Breast Cancer: Recommendations from the International Ki67 in Breast Cancer Working Group. J. Natl. Cancer Inst. 2011, 103, 1656-1664. [CrossRef]

72. Gerdes, J.; Lemke, H.; Baisch, H.; Wacker, H.H.; Schwab, U.; Stein, H. Cell cycle analysis of a cell proliferation-associated human nuclear antigen defined by the monoclonal antibody Ki-67. J. Immunol. 1984, 133, 1710-1715.

73. Thomas, S.; Johannes, G. The ki-67 protein: From the known and the unknown. J. Cell. Physiol. 2000, 182, 311-322.

74. Munn, Z.; Peters, M.D.J.; Stern, C.; Tufanaru, C.; McArthur, A.; Aromataris, E. Systematic review or scoping review? Guidance for authors when choosing between a systematic or scoping review approach. BMC Med. Res. Methodol. 2018, 18, 143. [CrossRef] [PubMed]

75. Gorjão, R.; Azevedo-Martins, A.K.; Rodrigues, H.G.; Abdulkader, F.; Arcisio-Miranda, M.; Procopio, J.; Curi, R. Comparative effects of DHA and EPA on cell function. Pharmacol. Ther. 2009, 122, 56-64. [CrossRef]

76. Serini, S.; Fasano, E.; Piccioni, E.; Cittadini, A.; Calviello, G. Differential Anti-Cancer Effects of Purified EPA and DHA and Possible Mechanisms Involved. Curr. Med. Chem. 2011, 18, 4065-4075. [CrossRef] [PubMed]

77. VanderSluis, L.; Mazurak, V.C.; Damaraju, S.; Field, C.J. Determination of the Relative Efficacy of Eicosapentaenoic Acid and Docosahexaenoic Acid for Anti-Cancer Effects in Human Breast Cancer Models. Int. J. Mol. Sci. 2017, 18, 2607. [CrossRef] [PubMed]

78. Dyall, S.C. Long-chain omega-3 fatty acids and the brain: A review of the independent and shared effects of EPA, DPA and DHA. Front. Aging Neurosci. 2015, 7, 52. [CrossRef]

79. Asztalos, I.B.; Gleason, J.A.; Sever, S.; Gedik, R.; Asztalos, B.F.; Horvath, K.V.; Dansinger, M.L.; Lamon-Fava, S.; Schaefer, E.J. Effects of eicosapentaenoic acid and docosahexaenoic acid on cardiovascular disease risk factors: A randomized clinical trial. Metabolism 2016, 65, 1636-1645. [CrossRef]

80. Berstad, P.; Thiis-Evensen, E.; Vatn, M.H.; Almendingen, K. Fatty Acids in Habitual Diet, Plasma Phospholipids, and Tumour and Normal Colonic Biopsies in Young Colorectal Cancer Patients. J. Oncol. 2012, 2012, 254801. [CrossRef] [PubMed]

81. Stillman, M.; Cata, J.P. Management of chemotherapy-induced peripheral neuropathy. Curr. Pain Headache Rep. 2006, 10, 279-287. [CrossRef]

82. Urruticoechea, A.; Smith, I.E.; Dowsett, M. Proliferation Marker Ki-67 in Early Breast Cancer. J. Clin. Oncol. 2005, 23, 7212-7220. [CrossRef]

83. Beuschlein, F.; Weigel, J.; Saeger, W.; Kroiss, M.; Wild, V.; Daffara, F.; Libé, R.; Ardito, A.; Al Ghuzlan, A.; Quinkler, M.; et al. Major Prognostic Role of Ki67 in Localized Adrenocortical Carcinoma after Complete Resection. J. Clin. Endocrinol. Metab. 2015, 100, 841-849. [CrossRef] [PubMed]

84. Powell, D.R.; Huttenlocher, A. Neutrophils in the Tumor Microenvironment. Trends Immunol. 2016, 37, 41-52. [CrossRef] [PubMed]

85. Macdonald, N. Cancer cachexia and targeting chronic inflammation: A unified approach to cancer treatment and palliative/supportive care. J. Support. Oncol. 2007, 5, 157-162. [PubMed] 